\title{
Interference Aspects of Adaptive Modems over Slow Rayleigh Fading Channels
}

\author{
Jeff M. Torrance, Lajos Hanzo, Senior Member, IEEE, and Thomas Keller
}

\begin{abstract}
Adaptive modulation can achieve channel capacity gains by adapting the number of bits per transmission symbol on a burst-by-burst basis, in harmony with channel quality fluctuations. This is demonstrated in the paper for target bit error rates of 1 and $0.01 \%$, respectively, in comparison to conventional fixed modems. However, the achievable gains depend strongly on the prevalant interference levels and hence interference cancellation is invoked on the basis of adjusting the demodulation decision boundaries after estimating the interfering channel's magnitude and phase. Using the modem-mode switching levels of Table $\mathrm{X}$ and with the aid of interference cancellation, target BER's of 1 and $0.01 \%$ can be maintained over slow-fading channels for a wide range of channel Signal-to-noise ratios (SNR) and Signal-tointerference ratios (SIR), as seen in Figs. 20 and 21, respectively.
\end{abstract}

Index Terms - Burst-by-burst adaptive modulation (modems), cochannel interference, interference cancellation.

\section{Adaptive Modulation}

B URST-BY-BURST adaptive multilevel modulation was first suggested in [1]-[3] for slowly fading wireless pedestrian channels, inspiring intensive further research in recent years [4]-[15]. The proposed schemes provide a means of realizing some of the time-variant channel capacity potential of the fading wireless channel [17], [19], invoking a more robust transmission scheme (TS) on a burst-by-burst basis, when the channel is of low quality and vice versa, while maintaining a certain target bit error rate (BER) performance. The most appropriate TS is dependent upon the time-variant instantaneous signal-to-noise ratio (SNR) and signal-to-interference ratio (SIR). The TS can be chosen according to the following regime [9]:

$$
\mathrm{TS}= \begin{cases}\text { No Transmission (Notx) } & \text { if } l_{1}>s^{2} / N \\ \text { BPSK } & \text { if } l_{1} \leq s^{2} / N<l_{2} \\ \text { QPSK } & \text { if } l_{2} \leq s^{2} / N<l_{3} \\ \text { Square 16-Point QAM } & \text { if } l_{3} \leq s^{2} / N<l_{4} \\ \text { Square 64-Point QAM } & \text { if } s^{2} / N \geq l_{4}\end{cases}
$$

where $s$ is the instantaneous signal level, $N$ is the average noise power, and $l_{1}, l_{2}, l_{3}$, and $l_{4}$, are the BER-dependent optimized switching SNR's. Time division duplex (TDD) was proposed, in order to exploit the reciprocity of the channel under high SIR conditions, which allowed us to estimate the

Manuscript received June 12, 1997; revised June 23, 1998. This work was supported by Motorola ECID, Swindon, U.K.; European Community, Brussels, Belgium; Engineering and Physical Sciences Research Council, Swindon, U.K.; and Mobile Virtual Centre of Excellence, U.K.

The authors are with the Department of Electrical and Computer Science, University of Southampton, Southampton, SO17 1BJ, U.K.

Publisher Item Identifier S 0018-9545(99)07387-9.
TABLE I

Switching Levels for Speech and Computer Data Systems Through a RaYleigh Channel, Shown in Instantaneous Channel SNR (dB) to Achieve Mean BER's of $1 \times 10^{-2}$ AND $1 \times 10^{-4}$, Respectively

\begin{tabular}{l|c|c|c|c}
\hline Switching levels(dB) & $l_{1}$ & $l_{2}$ & $l_{3}$ & $l_{4}$ \\
\hline Mean-Speech (1\%) & 3.31 & 6.48 & 11.61 & 17.64 \\
Mean-BER Data (0.01\%) & 7.98 & 10.42 & 16.76 & 26.33 \\
\hline
\end{tabular}

prevalent SNR on a burst-by-burst basis [10]. The reciprocity of the up- and downlink channel conditions in the TDD frame is best approximated, if the corresponding TDD slots are adjacent.

However, moving the associated duplex up- and downlink slots closer together extends the time duration measured from the downlink slot to the next uplink slot, degrading the validity of the channel's reciprocity in this context. In order to circumvent this deficiency we proposed to "passively" estimate the channel quality of the TDD slot prior to the mobile's uplink slot, whithout demodulating it. The uplink TS is chosen on the basis of this channel quality estimate, etc. In [9], we have characterized the analytical upper-bound performance of such a scheme for slow Rayleigh fading channels, while in [12] we introduced an unequal protection phasor constellation for signaling the current TS. The problem of appropriate power assignment was discussed, for example, in [14] and [6].

In [11] a combined BER- and BPS-based optimization costfunction was defined and minimized, in order to find the required TS switching levels for maintaining average target BER's of $1 \times 10^{-2}$ and $1 \times 10^{-4}$, irrespective of the instantaneous channel SNR. We referred to the former scheme as the speech TS, while to the latter as the adaptive data TS. The above BER's are sufficiently low for error-correction codecs to reduce these values further, in order to maintain typical wireless speech- and data-service qualities. The optimized TS switching levels $l_{1}, l_{2}, l_{3}$, and $l_{4}$ are summarized in Table I [9]. The average BPS performance $B$ of our adaptive modem was derived for a Rayleigh fading channel in [9], which can be written as

$$
\begin{aligned}
B= & 1 \cdot \int_{l_{1}}^{l_{2}} F(l, L) d l+2 \cdot \int_{l_{2}}^{l_{3}} F(l, L) d l \\
& +4 \cdot \int_{l_{3}}^{l_{4}} F(l, L) d l+6 \cdot \int_{l_{4}}^{\infty} F(l, L) d l
\end{aligned}
$$

where

$$
F(l, L)=\frac{1}{L} \cdot e^{-l / L}, \quad l \geq 0
$$


TABLE II

aTdMa Cell Types and Modulation Schemes, Note That Symbol Rates Refer to Offwset Rates Which Are Twice as High as the Equivalent Nonoffset Modem Discussed in This PaPer

\begin{tabular}{|c|c|c|c|c|c|c|c|c|}
\hline & \multicolumn{4}{|c|}{ Speech } & \multicolumn{4}{|c|}{ Computer Data } \\
\hline $\operatorname{SIR}(\mathrm{dB})$ & $l_{1}$ & $l_{2}$ & $l_{3}$ & $l_{4}$ & $l_{1}$ & $l_{2}$ & $l_{3}$ & $l_{4}$ \\
\hline 10 & 4 & 10 & 27 & 35 & \multicolumn{4}{|c|}{ Unused } \\
\hline 20 & 3 & 6 & 12 & 30 & 14 & 30 & 38 & 60 \\
\hline 30 & 3 & 6 & 12 & 18 & 8 & 11 & 17 & 60 \\
\hline 40 & 3 & 6 & 12 & 18 & 8 & 11 & 17 & 25 \\
\hline
\end{tabular}

is the probability density function (pdf) of the received SNR $l=s^{2} / N$ and $L=S / N$ is the average received SNR, if the instantaneous signal amplitude $s$ is Rayleigh distributed, obeying

$$
F(s, S)=\frac{2 s}{S} \cdot e^{-s^{2} / S}, \quad s \geq 0
$$

while $S$ is the average signal power. The individual terms constituted by integrals characterize the received signal level domains, where the 1, 2, 4, and 6 bits/symbol TS's of (1) are used. In [15] and [16], the latency performance of these schemes was quantified and frequency hopping as well as statistical multiplexing were proposed to mitigate its latency and buffer requirements.

In this contribution we focused our attention on the optimum choice of adaptive modem switching levels required to maintain target BER's of 1 and $0.01 \%$, respectively, under interfered conditions. Our experiments were conducted within the framework of the advanced time-division multipleaccess (ATDMA) [21], [22] scheme, for which the cell types and modulation schemes are summarized in Table II. In our cochannel interference investigations the signal was transmitted through a $10-\mathrm{ms}^{-1}$ vehicular-speed Rayleigh channel and the interference was faded through an independent $1-\mathrm{ms}^{-1}$ channel.

Following a brief discussion on the effects of adjacent and cochannel interference (CCI) in Section II, the CCI performance of adaptive modems is quantified in Section III. An interference cancellation scheme and its performance are characterized in Section IV, while the channel capacity of the proposed adaptive schemes is analyzed in Section V. We offer some concluding remarks in Section VI and some hints on our future work.

\section{INTERFERENCE RESISTANCE OF CONVENTIONAL MODEMS OVER RAYLEIGH CHANNELS}

In this section, we initially quantify the interference resistance of conventional time-invariant modems in a TDD scenario under the worst case conditions, where both the signal and interferer are exposed to Rayleigh fading. There is no evidence to support any significant correlation between the Doppler frequencies of the wanted and interfering signals. The only exception to this is during the downlink, where the correlation bandwidth is wide enough, and hence two channels adjacent in frequency and in the equivalent time slots could have fading with the same Doppler frequencies. This would result in the generally favorable situation where high interference levels are temporally correlated with high

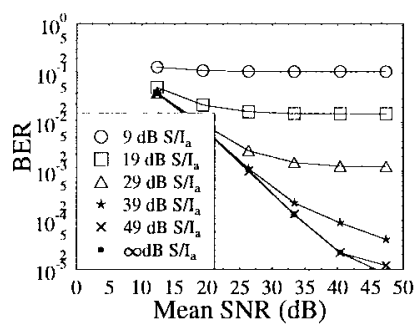

(a)

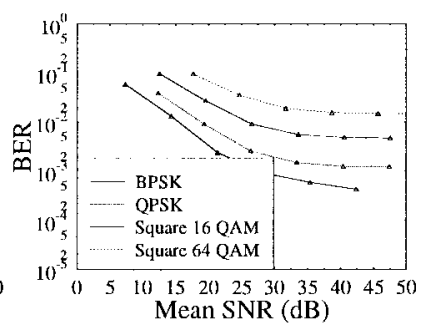

(b)
Fig. 1. BER performance of various modulation schemes through a Rayleigh channel with Rayleigh interference.

received-signal levels. This situation is not representative and therefore no general correlation is expected between the interference and signal levels.

In order to evaluate the performance of BPSK, QPSK, and square 16- and 64-QAM in Rayleigh channels with single Rayleigh interferers, a series of simulations was conducted. To ensure that the interference levels were uncorrelated with the signal levels, channels exhibiting different Doppler frequencies were used for the signal and interferer. However, using a channel with the same Doppler frequency for the signal and the single interferer gave comparable results to using different Doppler frequencies, if there was sufficient temporal decoupling between the fading of the signal and that of the interference. The experiments were conducted for SNR values between $0-50 \mathrm{~dB}$, and the range of SIR values encompassed 9, 19, and $29 \mathrm{~dB}$ for BPSK, 9, 19, 29, 39, and $49 \mathrm{~dB}$ for QPSK, 19,29 , and $39 \mathrm{~dB}$ for square 16-QAM and 29, 39, and 49 $\mathrm{dB}$ for square 64-QAM. Adjacent and cochannel interference were investigated in isolation and in every case the signal was transmitted through a $10-\mathrm{m} / \mathrm{s}$ vehicular-speed Rayleigh channel, while the interference was faded employing a $1-\mathrm{m} / \mathrm{s}$ channel. An example of the results is shown in Fig. 1.

Fig. 1(a) shows the performance of QPSK through a Rayleigh channel with Rayleigh fading adjacent channel interference (ACI). The results for identical levels of cochannel interference $(\mathrm{CCI})$ were indistinguishable from these performance curves. The BER performance is independent of the type of interference in the Rayleigh case, because the fluctuation in signal and interference is so large that the fine structure of the interference becomes insignificant. It can be seen from Fig. 1(a) that for all signal-to-adjacent channel interference ratios $S / I_{a}$ (and, therefore, also for the same cochannel interference ratios $S / I_{c}$ ) there is some observable residual BER. Fig. 1(b) shows the performance for all the schemes at $29 \mathrm{~dB} S / I_{a}$, which also exhibits a residual BER. Table III displays the residual BER's, and the SNR's at which they occur for various modulation schemes with various levels of ACI and CCI.

In a practical system there will be combinations of coand/or adjacent-channel interferers. Multiple interferers will result in both the ACI and CCI becoming more noise-like due to the central limit theorem, exhibiting a Gaussian-like pdf. Hence, for a given SIR with six interferers, as proposed by Lee [17], the performance degradation is likely to be less dramatic than introduced by a single interferer with the same SIR, which 
TABLE III

SNR AND BER VALuEs of Residual BER for VARIOUs Modulation Schemes in a Rayleigh Channel. The Interference Was Simulated Using QPSK, a NoRMalized AdJacent CARRIER SEPARATION OF $\Delta f \cdot T=0.23$, AND A NyQUist RollofF FACTOR OF $\alpha=0.35$. THE $\times$ REFLECTS That $50 \mathrm{~dB}$ WAS AN INSUFFICIENT SNR to IDENTIFY a RESIDUAL BER, AND THE - REFlects That No ExPERIMENT Was CONDUCTED

\begin{tabular}{|c|c|c|c|c|}
\hline$S / I_{a}$ & BPSK & QPSK & QAM 16 & QAM 64 \\
\hline 9 & $27 \mathrm{~dB}, 7 \%$ & $27 \mathrm{~dB}, 10 \%$ & & \\
\hline 19 & $37 \mathrm{~dB}, 0.8 \%$ & $34 \mathrm{~dB}, 1.5 \%$ & & \\
\hline 29 & $>50 \mathrm{~dB}, \approx 0.02 \%$ & $41 \mathrm{~dB}, 0.12 \%$ & $42 \mathrm{~dB}, 0.45 \%$ & $47 \mathrm{~dB}, 1.3 \%$ \\
\hline $\begin{array}{l}39 \\
49\end{array}$ & & $>50 \mathrm{~dB} \underset{\times}{\approx} \underset{x}{\approx} 0.003 \%$ & $\begin{array}{c}>50 \mathrm{~dB}, \approx 0.025 \% \\
\times\end{array}$ & $\begin{array}{c}>50 \mathrm{~dB}, \approx 0.15 \% \\
\times\end{array}$ \\
\hline
\end{tabular}

is a consequence of the less Gaussian-like interference pdf. Hence, the experimental results documented here represent the worst case interference scenario.

\section{THE EFFECTS OF MUlTiUsER INTERFERENCE ON ADAPTIVE MODULATION SCHEMES}

The effect of cochannel interference (CCI) upon our specific adaptive modulation scheme is potentially more detrimental than for a fixed modulation scheme. The reason for this is that the interference at the base station (BS) and mobile station (MS) is uncorrelated. Therefore, the interference corrupts not only the received symbols, but also adversely affects the TDDbased estimation of the channel quality. This is not necessarily a generic impediment of adaptive QAM (AQAM), since it is possible to invoke other solutions for jointly estimating both the SNR and SIR of the channel, but it is characteristic of our current regime. Furthermore, it would also be possible to refrain from estimating the expected channel quality upon assuming the channel's reciprocity and, instead, to adapt the modulation scheme on the basis of the signal-to-interefernceplus-noise ratio (SINR) on the previous transmission burst of the same link. ${ }^{1}$ However, the estimation of the prevalant SIR's and SINR is left for further study, following for example the approach of Andersin [20]. Below the effects of interference on the channel quality estimation and on the received signal quality itself are initially considered separately.

\section{A. Impact of Interference Upon Channel Estimate}

The lack of correlation between the up- and downlink interference is illustrated in Fig. 2. Here, it can be seen that although the channel characteristics of path $A$ (MS to BS) will approximate those of path $B$ (BS to MS), the interference from paths $C$ (interfering $\mathrm{MS}$ to $\mathrm{BS}$ ) and $D$ (interfering $\mathrm{BS}$ to MS) could be very different. This is because the average signal strength received from the interfering BS and interfering MS may vary considerably. Furthermore, even if transmission along each path resulted in the same received average signal strength, both paths will be fading independently.

It is assumed that for a given SIR value the statistical characteristics of the interference upon the uplink signal are the same as those on the downlink. This permits the analysis of only the downlink BER without loss of generality. The effects of frequency hopping (FH), voice activity detection (VAD), directional antennas, and power control upon the impact of

\footnotetext{
${ }^{1}$ The comments of the anonymous reviewer concerning this issue are gratefully acknowledged.
}

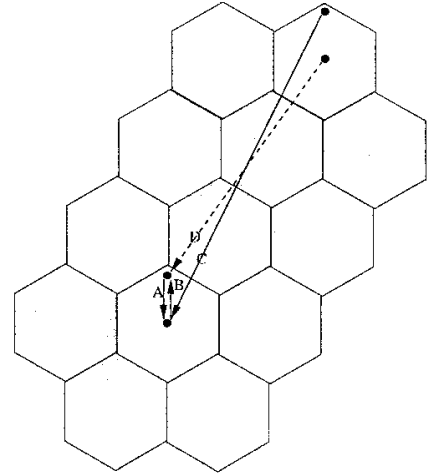

Fig. 2. The path $A$ is equivalent to $B$, however, the interference $D$ combined with the signal $B$ and the interference $A$ combined with the signal $C$ reduce this equivalence.

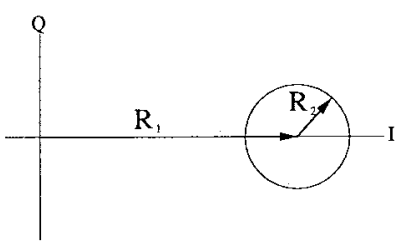

(a)

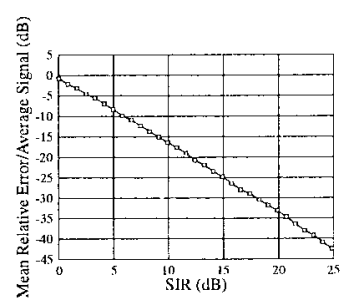

(b)
Fig. 3. Model and results of impact of interference upon channel estimate.

interference-corrupted channel estimates are left for further study.

Fig. 3(a) shows how the interference effects upon the estimation of the channel may be modeled. The vector $R_{1}$ represents the received signal vector, which in the absence of noise and interference would result in $\left|R_{1}\right|$ being a perfect estimation of the channel's amplitude gain. However, the signal is corrupted by interference and hence the channel amplitude gain is estimated as $\left|R_{1}+R_{2}\right|$ and, therefore, the error in the channel estimation is given by $\left|R_{1}+R_{2}\right|-\left|R_{1}\right|$. Clearly, the error is most critical when $\left|R_{1}\right|$ is small. Hence, the relative channel estimation error is defined as $\left(\left|R_{1}+R_{2}\right|-\left|R_{1}\right|\right) /\left|R_{1}\right|$. Mathematical analysis of the relative error is complex, given that $\left|R_{1}\right|$ and $\left|R_{2}\right|$ are Rayleigh distributed and $\left|R_{2}\right|$ has random uniform phase. A simplification can be achieved by assuming that $\left|R_{1}\right| \gg\left|R_{2}\right|$, where upon the error becomes Gaussian distributed, however, analysis is unnecessary, as it will be shown later. Furthermore, simulation of the relative channel estimation error versus SIR performance is trivial, which is evidenced by Fig. 3(b).

Fig. 3(b) shows that although large relative errors in the channel gain estimation will be inflicted by high-level Rayleigh fading interference, increased SIR results in considerable improvements in the relative error. This may results in overestimating the received signal level and hence invoking an optimistically high-order constellation for the downlink, but only if the MS's reception is also contaminated by interference-a condition which is independent of the interference perceived by the BS. 


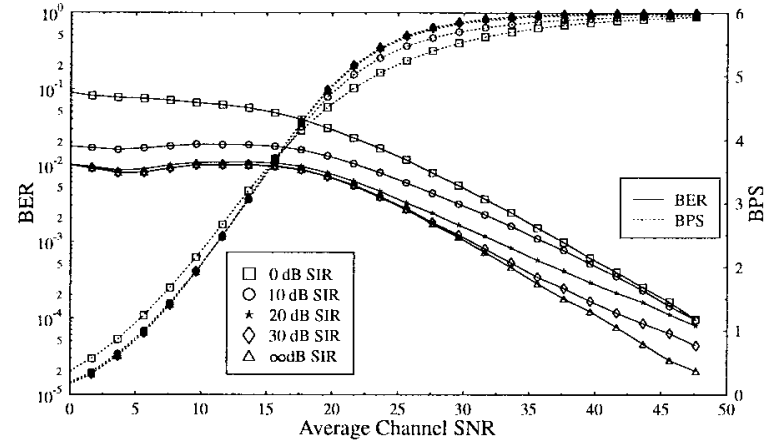

Fig. 4. Downlink BER over a slow Rayleigh fading channel with $\infty$ dB SIR at the MS and independent Rayleigh fading interference at the BS with SIR's of $0,10,20,30$, and $\infty$ dB with adaptive speech system mean BER switching levels. The interference at the BS affects only the channel-quality estimates.

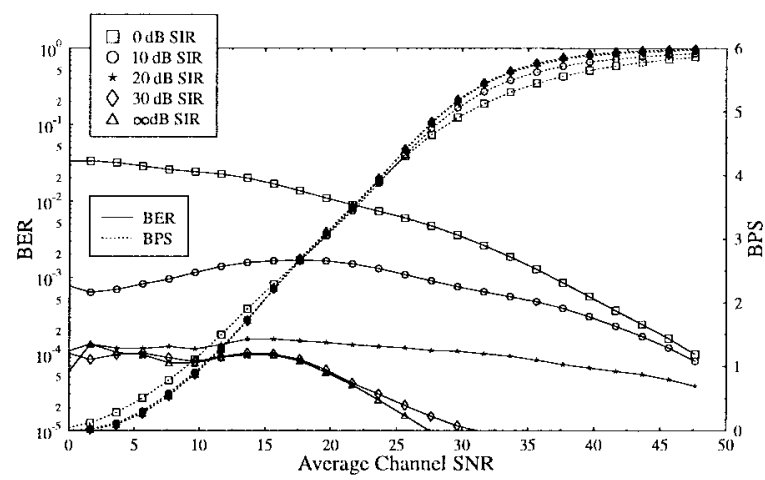

Fig. 5. Downlink BER over a slow Rayleigh fading channel with $\infty$ dB SIR at the MS and independent Rayleigh fading interference at the BS with SIR's of $0,10,20,30$, and $\infty$ dB with adaptive computer-data system mean BER switching levels. The interference at the BS affect only the channel-quality estimates.

Considering this hypothesis, experiments were conducted, which considered the downlink BER over a Rayleigh fading channel with $\infty \mathrm{dB}$ SIR at the mobile station. The channel was assumed to fade slowly and the estimates of the channel gain made at the base station were performed in the presence of a single independent Rayleigh fading interferer. The experiments were conducted with SIR's of $0,10,20,30$, and $\infty \mathrm{dB}$ for both the speech optimized switching levels and the computer data optimized switching levels.

The results are shown in Figs. 4 and 5. There are two types of curves in the figures, namely, the bit-error rate (BER) and the bits-per-symbol (BPS) curves, which are scaled on the left and right vertical axes, respectively. Let us initially consider the interference-free curves associated with an SIR of $\infty$. As mentioned in the Section I, we contrived a $1 \%$ BER adaptive speech system and a higher integrity $0.01 \%$ BER data system. The adaptive modulation scheme switching levels were optimized by Powell's optimization technique [18] or maintaining these target BER's over fading channels. As the average channel SNR improved, the adaptive modem was able to employ higher order constellations and this improved the average BPS modem performance. For high average SNR's, however, the BPS performance saturated at 6 bits/symbol and the BER curves decayed below the target BER's, since for reasons of moderate complexity the adaptive scheme was unable to opt for more than 6-bits/symbol constellations.

From Figs. 4 and 5 we infer that as the uplink SIR increases, the BER reduces at all SNR's. Observe that the computer data system is most sensitive to uplink interference with several orders of magnitude BER performance degradation, for all SNR's of interest, at 0-dB SIR and at least one order of magnitude BER performance degradation, for all SNR's of interest, at 10-dB SIR. The maximum BER performance degradation with the speech system is one order of magnitude at 0-dB SIR and at lower SNR's the degradation is less than a factor of two.

The consequence of uplink interference on the BPS performance is less dramatic than on the BER performance, as evidenced by Figs. 4 and 5. However, for both systems at low SIR and SNR, the average throughput is mistakenly increased due to the interference-boosted received signal levels and, therefore, less robust modulation schemes are employed more often than expected with the given (optimized) switching levels, if the uplink SIR was infinite. This is an explanation for the increased BER, when both the SIR and SNR are low for the speech and computer data schemes.

Note in Figs. 4 and 5 that at low SIR's and high SNR's the average BPS performance is reduced, implying that more robust modulation schemes are employed more often in comparison to when the uplink SIR is infinite. At first sight, this reduction in BPS performance is not consistent with the reduced BER performance, i.e., higher BER's, observed under such conditions. However, although the average throughput is reduced, and on average more robust modulation schemes are employed, the interference results in the base station failing to identify the depth of some fades and hence employing a higher order modulation scheme with insufficient protection. This generates small bursts of errors that increase the average BER. These error bursts have less significance, when the average BER is relatively high. Therefore, at the SNR's shown in the figures, the detrimental effect of reduced uplink SIR's in the computer data system is more noticeable than in the speech system.

The preceding discussions can be summarized by stating that the adaptive modulation's downlink performance is degraded, even in the absence of interference at the mobile, by interference at the base station, because the uplink interference results in the channel estimation being corrupted and a suboptimum modulation scheme being employed.

There are two modes of this misestimation of the channel amplitude gain, which will be discussed here. Considering the model of the interference presented in Fig. 3(a), the two modes correspond to the cases when the condition $\left|R_{1}\right| \gg\left|R_{2}\right|$ is met and when it is not. In the former case, the Rayleigh faded uplink interference results in an approximately equal probability of over- and underestimation of the channel amplitude gain. This is because the component of $\left|R_{2}\right|$ perpendicular to $\left|R_{1}\right|$ becomes negligible. Figs. 4 and 5 reveal that the $0-\mathrm{dB}$ SIR BPS curve behaves differently from the 10-, 20-, 30-, and $\infty$ dB SIR curves by not converging with the others at low SNR's. Therefore, it is concluded that the condition $\left|R_{1}\right| \gg\left|R_{2}\right|$ is not true for 0-dB SIR, but it holds for 10-dB SIR. 
TABLE IV

BPS Performance Versus SNR and SIR Extracted From Fig. 4

\begin{tabular}{l|c|c|c|c}
\hline & $0 \mathrm{~dB}$ SIR & $10 \mathrm{~dB}$ SIR & $20 \mathrm{~dB}$ SIR & $30 \mathrm{~dB}$ SIR \\
\hline$-0.36 \mathrm{~dB}$ SNR & $0 . .324$ & 0.153 & 0.131 & 0.129 \\
$1.64 \mathrm{~dB}$ SNR & 0.561 & 0.354 & 0.323 & 0.320 \\
$3.64 \mathrm{~dB}$ SNR & 0.866 & 0.640 & 0.606 & 0.603 \\
$\mathbf{5 . 6 4} \mathrm{dB}$ SNR & 1.243 & 1.000 & 0.967 & 0.963 \\
$7.64 \mathrm{~dB}$ SNR & 1.676 & 1.439 & 1.412 & 1.408 \\
\hline
\end{tabular}

TABLE V

BPS Performance Versus SNR and SIR Extracted From Fig. 5

\begin{tabular}{l|c|c|c|c}
\hline & $0 \mathrm{~dB}$ SIR & $10 \mathrm{~dB}$ SIR & $20 \mathrm{~dB}$ SIR & $30 \mathrm{~dB}$ SIR \\
\hline$-0.36 \mathrm{~dB}$ SNR & 0.033 & 0.003 & 0.002 & 0.002 \\
$1.64 \mathrm{~dB}$ SNR & 0.120 & 0.028 & 0.021 & 0.020 \\
$\mathbf{3 . 6 4} \mathrm{dB}$ SNR & 0.284 & 0.116 & 0.097 & 0.095 \\
$5.64 \mathrm{~dB}$ SNR & 0.512 & 0.304 & 0.272 & 0.270 \\
$7.64 \mathrm{~dB}$ SNR & 0.787 & 0.583 & 0.550 & 0.546 \\
\hline
\end{tabular}

Considering SIR's of $10 \mathrm{~dB}$ or higher and the pdf's of the individual modulation schemes employed for the speech and computer data systems, which were plotted in [13], it can be seen at low SNR's that misestimation of the channel amplitude gain results in an increase in the average BPS and at high average SNR's the reverse it true. This is consistent with the evidence in Figs. 4 and 5 for high average channel SNR's. For low average channel SNR's the figures do not show the effect well, however, a tabulation of the average BPS results is shown for the lower SNR values in Tables IV and V, which show the expected increase in average BPS at low SNR, as the SIR reduces.

The effect of $0-\mathrm{dB}$ SIR on the channel amplitude gain is more complex than at higher SIR's, but these effects are relatively unimportant, because the following work will not propose adaptive modulation at such low SIR's. The effects of uplink interference on the performance of adaptive modulation have been discussed. However, in terms of the design of the adaptive speech and data modulation schemes with BER's of $1 \times 10^{-2}$ and $1 \times 10^{-4}$ it may be concluded that the average uplink SIR must be 10 and $20 \mathrm{~dB}$, or above, for the respective systems, in order to maintain the required target BER's. Furthermore, it transpires from the previous discussion that the adaptive switching levels optimized for noninterfered channels have to be reconsidered in order to account for the effects of interference.

\section{B. Reoptimization of Adaptive Switching Levels}

As in the previous section, only the performance of the downlink was considered. However, it is assumed that the performance of the uplink would be identical, provided that the assumptions made about downlink interference applied to the uplink and vice versa. The effect of only downlink interference, i.e., interference at the MS only, is shown in Figs. 6 and 7 for the speech and computer data systems, respectively. That is, in contrast to our previous experiments, where the uplink interference corrupted the channel quality estimates, in these experiments the effect of the interference upon the channel estimate is neglected. For both the speech and computer data systems we found that the throughput was unaffected by the interference inflicted at the MS, since the channel-quality estimates are unaffected. This was anticipated, since the decision upon which the modulation scheme should

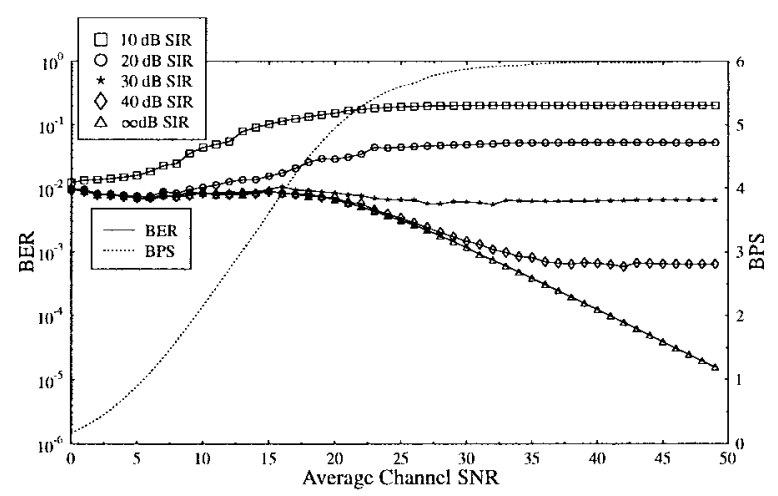

Fig. 6. Downlink BER over a slow Rayleigh fading channel with 10-, 20-, 30-, 40-, and $\infty-\mathrm{dB}$ SIR at the MS and no interference at the BS with the adaptive speech system mean BER switching levels, $\alpha=0.35$ and no interference cancellation.

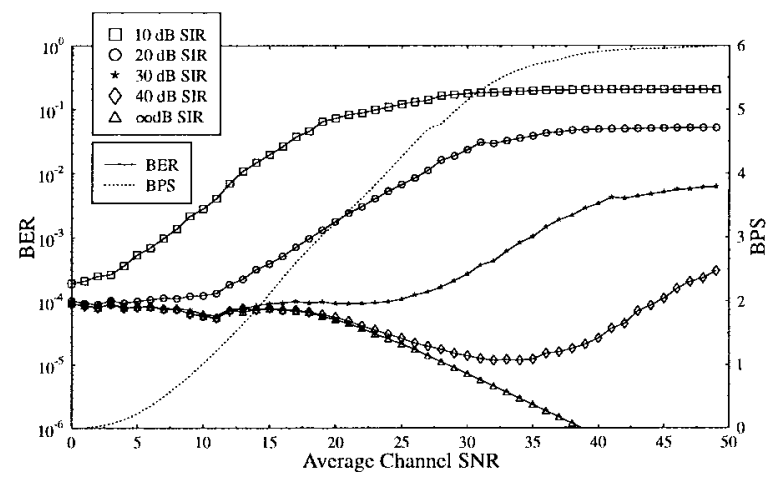

Fig. 7. Downlink BER over a slow Rayleigh fading channel with 10-, 20-, 30-, 40-, and $\infty-\mathrm{dB}$ SIR at the MS and no interference at the BS with adaptive computer data system mean BER switching levels, $\alpha=0.35$ and no interference cancellation.

be employed is made at the base station, which is unaffected by the interference experienced by the mobile station.

However, the BER was increased considerably for both the speech and computer data systems, as the SIR reduced, since the MS' effective experienced channel quality was reduced. In all cases except the speech system at 30- and 40-dB SIR, the introduction of cochannel interference at the MS resulted in an increased BER, as the average channel SNR increased. The explanation for this is that at higher average channel SNR's the receiver is led to believe that a high channel quality prevails and hence the probability of a higher order modulation scheme being employed increases, as was the case in the absence of interference. However, these high-order modulation schemes are more susceptible to interference as well as to noise. Therefore, bearing in mind that the switching levels were optimized in the absence of interference, it is clear that the BER is increased.

A logical approach to overcoming the interference would be to back off the switching levels at which the higher order modulation schemes should be employed. Such a technique would require an outer loop to identify the level of average interference and select a different set of switching levels accordingly. This could be achieved by considering the average received signal strength and comparing it with some informa- 
tion about the quality of the reception. This quality measure could be obtained from either channel coding overload rate or soft-decision information about the distance between received symbols and the ideal modulation constellation points. In this paper we opted to employ different switching levels when the average interference varied by $10 \mathrm{~dB}$, noting that the estimation of the prevalent SIR was left for future study, employing the solutions proposed in [20].

In order to obtain the modified TS switching levels in the presence of interference, the optimization algorithm described in [9] was employed again. Specifically, the BER and BPS performances were evaluated for average channel SNR's in the range of $0-50 \mathrm{~dB}$ in $1-\mathrm{dB}$ intervals, using the optimization cost function defined as

$$
\text { Total Cost }=\sum_{i=0}^{50} \text { BER Cost }(i)+\text { BPS Cost }(i)
$$

where

$$
\begin{aligned}
& \text { BER Cost }(i) \\
& \quad= \begin{cases}10 \log _{10}\left(\frac{\mathrm{BER}_{m}(i)}{\mathrm{BER}_{d}(i)}\right), & \text { if } \mathrm{BER}_{m}(i)>\mathrm{BER}_{d}(i) \\
0, & \text { otherwise }\end{cases}
\end{aligned}
$$

BPS $\operatorname{Cost}(i)$

$$
= \begin{cases}\mathrm{BPS}_{d}(i)-\mathrm{BPS}_{m}(i), & \text { if } \mathrm{BPS}_{d}(i)>\mathrm{BPS}_{m}(i) \\ 0, & \text { otherwise }\end{cases}
$$

and $\mathrm{BER}_{m}(i), \mathrm{BER}_{d}(i), \mathrm{BPS}_{m}(i)$, and $\mathrm{BPS}_{d}(i)$ are, respectively, the measured and desired BER and BPS at an average channel SNR of $i$. It can be seen from (5)-(7) that the cost function can only be positive and increases, when either the BER or the BPS performance become inferior to their desired performance at an average channel SNR of $i$. Equation (6) utilizes the logarithm function to increase the significance of small BER's. A weighting factor of 10 is employed in order to bias the optimization toward achieving the desired BER performance in preference to the BPS performance. Under interfered conditions the cost function of (7) was slightly modified to

$$
\begin{aligned}
& \text { BPS Cost }(i) \\
& \begin{cases}5 \cdot\left(\mathrm{BPS}_{d}(i)-\mathrm{BPS}_{m}(i)\right), & \text { if } \mathrm{BPS}_{d}(i)>\mathrm{BPS}_{m}(i) \\
0, & \text { otherwise. }\end{cases}
\end{aligned}
$$

This increased the weighting toward achieving the desired BPS performance at the cost of the desired BER performance. It was necessary to invoke this modification, since otherwise due to the presence of high interference levels the optimization total cost given by (5) was minimized, when the no-transmit mode was employed for all signal levels.

Reoptimized switching levels were derived for both the speech and computer data systems, where the desired performances were the same as before, that is, a speech system with average BER and BPS performance targets of $1 \times 10^{-2}$ and 4.5 bits/symbol, respectively, and a computer data system with average BER and BPS performance figures of $1 \times 10^{-4}$
TABLE VI

Reoptimized Switching Levels For Speech System Through a RAYLEIGH CHANNEL WITH INDEPENDENT RAYLEIGH INTERFERENCE AT DifFerent Average SIR's; Switching Levels Shown IN dB SNR

\begin{tabular}{l|c|c|c|c}
\hline Scheme & $l_{1}$ & $l_{2}$ & $l_{3}$ & $l_{4}$ \\
\hline $10 \mathrm{~dB}$ SIR & 4.06 & 9.37 & 14.05 & 18.38 \\
$20 \mathrm{~dB}$ SIR & 3.02 & 7.07 & 11.59 & 44.38 \\
$30 \mathrm{~dB}$ SIR & 2.98 & 6.48 & 11.60 & 17.64 \\
$40 \mathrm{~dB}$ SIR & 2.33 & 6.55 & 11.33 & 17.36 \\
\hline
\end{tabular}

TABLE VII

Reoptimized Switching Levels for Computer Data System Through a RAYLEIGH CHANNEL WITH INDEPENDENT RAYLEIGH INTERFERENCE AT DifFERENT Average SIR's; Switching Levels ShOwn IN dB SNR

\begin{tabular}{l|c|c|c|c}
\hline Scheme & $l_{1}$ & $l_{2}$ & $l_{3}$ & $l_{4}$ \\
\hline $20 \mathrm{~dB}$ SIR & 30.13 & 40.37 & 42.92 & 72.99 \\
$30 \mathrm{~dB}$ SIR & 7.93 & 11.22 & 32.14 & 102.81 \\
$40 \mathrm{~dB}$ SIR & 7.88 & 10.42 & 17.44 & 53.41 \\
\hline
\end{tabular}

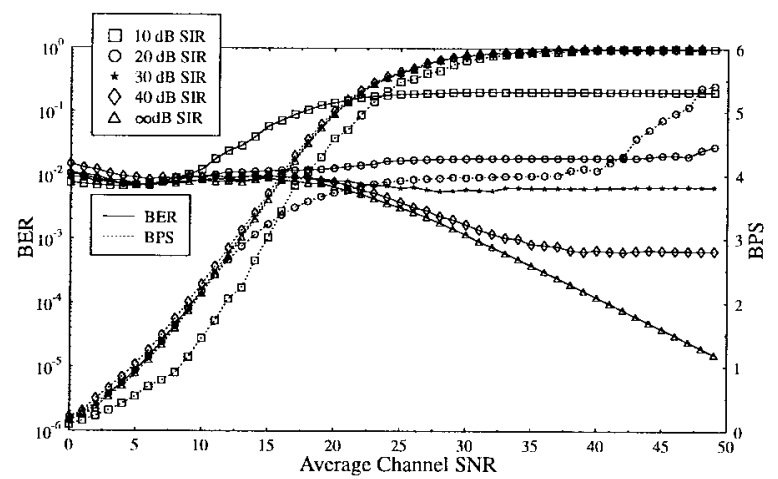

Fig. 8. Downlink BER over a slow Rayleigh fading channel with 10, 20-, $30-, 40-$, and $\infty \mathrm{dB}$ SIR at the MS and no interference at the BS and adaptive modulation with switching levels reoptimized for each SIR. The desired performance was BER $1 \times 10^{-2}$ and 4.5 BPS, $\alpha=0.35$ and no interference cancellation.

and 3 bits/symbol, respectively. For the speech system the switching levels were reoptimized for 10-, 20-, 30-, and 40$\mathrm{dB}$ SIR and for the computer data system they were optimized for 20-, 30-, and 40-dB SIR. No switching levels were derived for the computer data system at 10-dB downlink SIR, because the reverse link channel estimation has already been shown unacceptable in Fig. 5 at this level of interference, when the desired BER is $1 \times 10^{-4}$. The initial switching levels that were used to start the reoptimization algorithm were the switching levels derived in the original optimization, at $\infty \mathrm{dB}$ SIR. These were the levels that are shown in Table I.

The reoptimized switching levels for the speech and computer data systems are shown in Tables VI and VII, respectively. The BER and BPS performance of both schemes with the reoptimized switching levels are shown in Figs. 8 and 9, where the original optimized schemes' performance at $\infty \mathrm{dB}$ are also included for comparison.

The reoptimized switching levels for the speech system are generally similar to the switching levels that were obtained with the original optimization at $\infty \mathrm{dB}$ SIR. The exceptions are the switching levels obtained with the reoptimization at 10-dB SIR, where all levels are generally $1-3 \mathrm{~dB}$ SNR higher than the original corresponding optimal levels, and $l_{4}$ for 


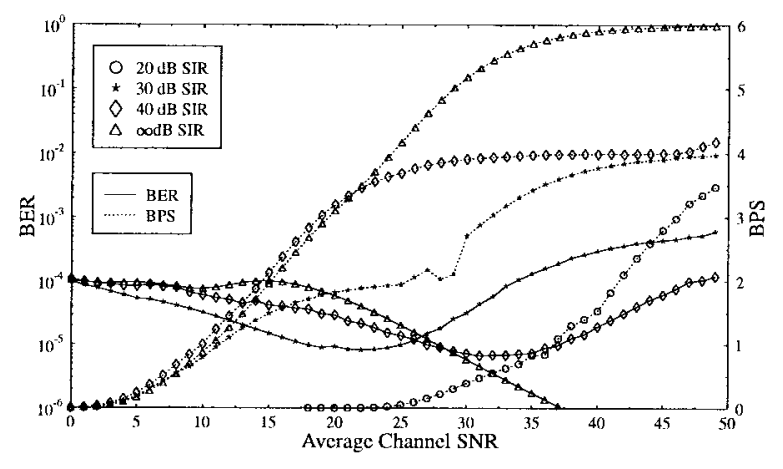

Fig. 9. Downlink BER over a slow Rayleigh fading channel with 20-, 30-, $40-$, and $\infty$-dB SIR at the MS and no interference at the BS and adaptive modulation with switching levels reoptimized for each SIR. The desired performance was BER $1 \times 10^{-4}$ and 3 BPS, $\alpha=0.35$ and no interference cancellation.

the reoptimization at $20-\mathrm{dB}$ SIR, which is approximately 27 $\mathrm{dB}$ higher than the original optimal level. This value of $l_{4}$ appears inconsistent compared with the $l_{4}$ values at other SIR's. However, at $10-\mathrm{dB}$ SIR $l_{4}>18.38$ would have had produced a greater increase in total cost, in terms of the cost increase associated with reducing the throughput, compared with the cost reduction registered from reducing the BER. This is because the square 64- and 16-QAM BER performance is similarly bad at $10-\mathrm{dB}$ SIR and consequently reducing $l_{4}$ will result in square 16-QAM being employed rather than square 64-QAM. Therefore, it can be observed, compared with the 20-dB SIR case, that the 10-dB SIR case benefits less from the reduction of the BER cost associated with reducing $l_{4}$, but it is penalized equally in terms of the increases in BPS cost associated with the same reduction of $l_{4}$. At 30-dB SIR there is no need to reduce $l_{4}$, because the target BER is achieved.

The effect of the significant changes in switching levels, and the more subtle changes can be identified in the performance curves. Inspection of the BPS performance curves shown in Fig. 8 reveals that the throughput for the reoptimized speech schemes at 20-, 30-, and 40-dB SIR closely coincided with the originally optimized scheme between 0 - and 12.5-dB average channel SNR. Above 12.5-dB average channel SNR the 20-dB SIR reoptimized throughput is lower than observed with the other schemes. This is the effect of the large value of $l_{4}$ in the $20-\mathrm{dB}$ reoptimized scheme. Furthermore, the throughput of the $10-\mathrm{dB}$ SIR reoptimized scheme is below the originally optimized scheme's throughput and again, this is an expected consequence of the increased value of the switching levels for the reoptimized scheme at $10-\mathrm{dB}$ SIR.

Considering the BER performance curves in Fig. 8 it may be observed that only the 30,40 , and $\infty$ dB SIR reoptimized switching levels result in the target BER being achieved for all average channel SNR's, at the respective interference levels. The 10- and 20-dB SIR reoptimized switching levels fail to achieve the target BER over the entire average channel SNR range. However, the same observation may be made about the originally intersymbol interference (ISI) free optimized switching levels and their BER performance which was shown in Fig. 6. It is not surprising that the 30- and 40-dB SIR reoptimized switching levels result in similar BER perfor- mance to the original optimized switching levels with 30and 40-dB SIR, because the switching levels are so similar. Furthermore, the reoptimization at these interference levels was unlikely to result in the switching levels being altered significantly, since the original switching levels already met the BER performance criteria. Reoptimizing the switching levels for 10- and 20- dB SIR resulted in significant changes in the switching levels, when compared with the original optimized levels. This change in switching levels reflects in the change in BER performance that can be observed by comparing Figs. 6 and 8. This comparison reveals that the reoptimization improved the BER performance at 10-dB SIR below an average channel SNR of $15 \mathrm{~dB}$ and at 20-dB SIR from 10-50-dB average channel SNR's. In the latter case, the effect was approximately a factor five BER reduction.

The reoptimized switching levels for the computer data system at 20-, 30-, and 40-dB SIR differ considerably more from the original $\infty-\mathrm{dB}$ SIR levels, than the speech switching levels at 20-, 30-, and 40-dB SIR do from their original $\infty-\mathrm{dB}$ SIR optimized switching levels. This is because the originally optimized switching levels for the computer data systm, when the SIR was $40 \mathrm{~dB}$ or less, resulted in a lower BER performance than that desired. Therefore, reoptimization resulted in the switching levels being modified. Employing the original computer data system switching levels, that were optimized for $\infty-\mathrm{dB}$ SIR, in interfered channels revealed that SIR's greater than $40 \mathrm{~dB}$ would be required to achieve the desired BER for average channel SNR's from 0-50 dB. The reoptimized switching levels result in the desired BER being achieved for $20-^{2}$ and $40-\mathrm{dB}$ SIR. Moreover, reoptimization at the specific SIR results in the performance of the reoptimized switching levels at $30 \mathrm{~dB}$ SIR approaching the desired BER performance.

The penalty of employing reoptimized switching levels, is as expected, a reduction in throughput. In the case of the BPS prformance of the switching levels reoptimized for 20dB SIR there is no throughput for average channel SNR's below $17 \mathrm{~dB}$ and, therefore, the BER performance below this level is uninteresting and the corresponding BER curve was omitted. The average throughput performance of the reoptimized switching levels at 20-dB SIR is approximately equivalent to BPSK at $40-\mathrm{dB}$ average channel SNR. The BER at this average SNR is less than $1 \times 10^{-6}$. In closing we note that we evaluated the fixed BPSK BER performance at the same average channel SNR, which was found to be 2.3 $\times 10^{-3}$. Following the above experiments we intuitively felt that further exploration of the switching-threshold-dependent tradeoffs are beneficial and we report on these endeavors in the next section.

1) Intuitive Threshold Adjustment: The reoptimized switching levels for the speech system in the presence of CCI are considered again. Since the target BER performance of $1 \%$ was not achieved for the 10 - and $20-\mathrm{dB}$ downlink SIR, there is some doubt over the suitability of the optimization algorithm or the definition of the cost function. Therefore,

\footnotetext{
${ }^{2}$ The 20-dB SIR reoptimized switching levels result in an extremely low BER with maximum value of $5.78 \times 10^{-10}$ at $50-\mathrm{dB}$ average channel SNR and the corresponding curve is therefore not plotted in Fig. 9.
} 


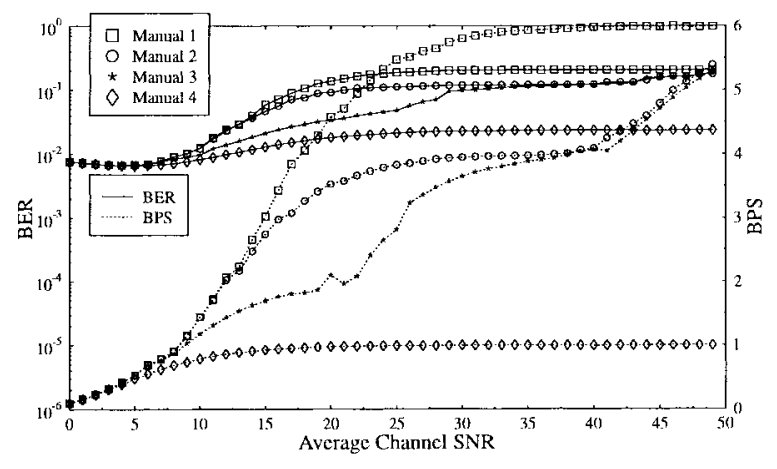

Fig. 10. Downlink BER over a slow Rayleigh fading channel with $\infty$-dB SIR at the base station and 10-dB SIR independent Rayleigh fading interference at the mobile-station using manually selected switching levels shown in Table VIII and $\alpha=0.35$.

TABLE VIII

Manually Selected Switching Levels Expressed IN SNR dB for Performance Curves IN Fig. 10

\begin{tabular}{l|c|c|c|c}
\hline Scheme & $l_{1}(\mathrm{~dB})$ & $l_{2}(\mathrm{~dB})$ & $l_{3}(\mathrm{~dB})$ & $l_{4}(\mathrm{~dB})$ \\
\hline Manual 1 & 4.06 & 9.37 & 14.05 & 18.38 \\
Manual 2 & 4.06 & 9.37 & 14.05 & 45.00 \\
Manual 3 & 4.06 & 9.37 & 25.00 & 45.00 \\
Manual 4 & 4.06 & 1000 & 1000 & 1000 \\
\hline
\end{tabular}

in an attempt to achieve the desired BER performance the reoptimized switching levels at 10-dB SIR were manually adjusted. Fig. 10 portrays the performance of the adaptive modem employing the manually adjusted switching levels and Table VIII shows a summary of the manually selected levels.

The set of switching levels, "Manual 1," are the values from the reoptimization that were given in Table VI at 10-dB SIR and are included for comparison. The set of "Manual 2" values reduces the employment of square 64-QAM by invoking it only at very high SNR's. Logically, this also reduces the average throughput for average channel SNR's higher than approximately $12 \mathrm{~dB}$. Square 64-QAM is the most corruptionsensitive modulation scheme and reducing its employment reduced the average BER. However, the performance at 10$\mathrm{dB}$ SIR is still worse than the target BER of $1 \%$ across the range of average channel SNR's. The "Manual 3" set of switching levels reduces the throughput further by reducing the employment of square 16-QAM. This again reduces the throughput, but does improve the BER mainly in the range of average channel SNR's from 10 to $30 \mathrm{~dB}$. However, the target BER performance is not achieved, especially at higher average channel SNR's.

In an extreme attempt to achieve the target BER at high average channel SNR's the switching levels "Manual 4" were evaluated. These essentially eliminated the employment of QPSK, and square 16- and 64-QAM. The resulting performance at high average channel SNR's is not surprising. The average BPS performance converges to the fixed BPSK performance, that is one BPS, and, although not shown due to lack of space, the BER performance converges with the fixed BPSK residual BER performance of $2.5 \times 10^{-2}$ at 10-dB SIR. Therefore, in order to reduce the BER below the BPSK residual, $l_{1}$ must be increased. This is undesirable, because at low average channel SNR the target BER is obtained with $l_{1}$ at its current value. Therefore, increasing $l_{1}$ extends the range of "No transmission" and hence will result in excessively low BER and a state of permanent "No transmission" at low average channel SNR's. This is similar to what was experienced, when $l_{1}$ became large in the $20-\mathrm{dB}$ SIR reoptimization for the computer data target system.

It can be concluded that switching threshold reoptimization, as described above, may be employed to reduce the margin between the desired BER and that achieved in the presence of interference. However, as a mechanism to overcome the effects of interference and achieve an arbitrary BER further techniques will be considered in the next section.

\section{INTERFERENCE CANCELLATION}

\section{A. Background}

Equalization is a well-established technique in cellular systems for reducing the effects of intersymbol interference (ISI). GSM [24], for example, incorporates a training sequence into a data burst, providing an estimate of the impulse response of the channel. This knowledge can be exploited to remove the intersymbol interference from the received sequence. The reduction of CCI may be incorporated into this equalization process, for example as proposed by Fukawa and Suzuki in [25]. Wales [26] recognized that if ISI cames from a single interferer [28], then the joint equalization and cochannel interference cancellation could be improved by acquiring information about the phase and amplitude of the cochannel interference propagation channel. He proposed obtaining information about the cochannel interference propagation channel by exploiting the orthogonality of the different training sequences. Murata $e t$ al. [29] considered exploiting the additional redundancy in both the signal and cochannel interferer, introduced by trellis coding, in order to improve the performance of an interference cancellation algorithm. However, they achieved this without an exponential growth in complexity that would typically be expected in such a system. Berangi et al. [30] showed up to a factor of 30 improvement in BER for narrowband constant amplitude-modulation schemes through Rayleigh fading channels.

Adaptive modulation is useful in combating the variation in received signal strength, and the consequential variation in SNR, which are encountered over fading mobile-radio channels. However, Section III-B1 revealed that adaptive modulation is vulnerable to CCI. Therefore, it is proposed to invoke some of the interference cancellation techniques that have recently been suggested, in combination with adaptive modulation, and therefore propose an overall adaptive transmission scheme that is resistant to noise and interference.

\section{B. Principle of Interference Cancellation}

The interference canceler's operation is essentially based on the ideas outlined by Wales in [26] and its concept is highlighted for the situation where a BPSK signal experiences CCI from a single BPSK interferer. The principle is readily extended for other scenarios. Consider the transmission of 
binary bits, at a rate of $T^{-1}$, where $b_{s}(n T)$ is the $n$th bit. This may be modulated as a stream of BPSK symbols. As a simplification, it will be assumed that the interference is phasenoncoherent and time-synchronous with the signal. Therefore, the transmitted symbols may be represented in the baseband by their value at the perfect sample position, namely by

$$
X_{s}(n T)= \begin{cases}+1+j 0, & \text { if } b_{s}(n T)=0 \\ -1+j 0, & \text { if } b_{s}(n T)=1\end{cases}
$$

assuming that the clock recovery will be perfect at the receiver. A single BPSK interferer's transmission may be represented by

$$
X_{i}(n T)= \begin{cases}+1+j 0, & \text { if } b_{i}(n T)=0 \\ -1+j 0, & \text { if } b_{i}(n T)=1\end{cases}
$$

The channel distortion introduced to $X_{s}(n T)$ and $X_{i}(n T)$ are respectively given by the complex variables $R_{s}(n T)$ and $R_{i}(n T)$. Therefore, the received signal is given by

$$
Y(n T)=R_{s}(n T) \cdot X_{s}(n T)+R_{i}(n T) \cdot X_{i}(n T)+N(t)
$$

where $N(t)$ is the complex Gaussian noise.

Restricting our investigations to narrowband channels, and initially assuming perfect knowledge of the complex channel gains $R_{s}(n T)$ and $R_{i}(n T)$, the receiver can determine which $X_{s}(n T)$ and $X_{i}(n T)$ symbols are most likely to have been transmitted on the basis of the received signal $Y(n T)$. This is simply achieved by determining the possible values of

$$
R_{s}(n T) \cdot X_{s}(n T)+R_{i}(n T) \cdot X_{i}(n T)
$$

and finding the value with the minimum Euclidean distance from $Y(n T)$. In the case of a BPSK signal and BPSK interference

$$
R_{s}(n T) \cdot X_{s}(n T)+R_{i}(n T) \cdot X_{i}(n T)
$$

has four possible values, assuming fixed values of $X_{s}(n T)$ and $X_{i}(n T)$. Without loss of generality we assume $R_{s}(n T)=$ $1+j 0$ for all $n$. Fig. 11 shows the four possible received points for arbitrary and equiprobable phase values of $\theta=$ $\pi / 3, \pi / 2,5 \pi / 6$, or $\pi$ where $\theta$ is the phase of $R_{i}(n T)$. This figure will be discussed in more detail in Section IV-C, however, it clearly shows that the decision boundaries vary with $\theta$.

\section{Fixed Schemes}

As stated above, the key motivation behind investigating interference cancellation is that it would appear to be an ideal technique to support adaptive modulation in a fading multiuser environment. However, in order to characterize the interference cancellation techniques and understand how they perform, they are initially considered in nonfading environments in conjunction with fixed-modulation schemes.

The performance of interference cancellation is relatively easily determined by simulation. However, analytical performance studies become increasingly difficult as the constellation size increases. The analysis is particularly difficult because, unlike the performance of square constellations in the presence of noise and interference, the acceptable corruption in the in-phase and quadrature components is not independent.

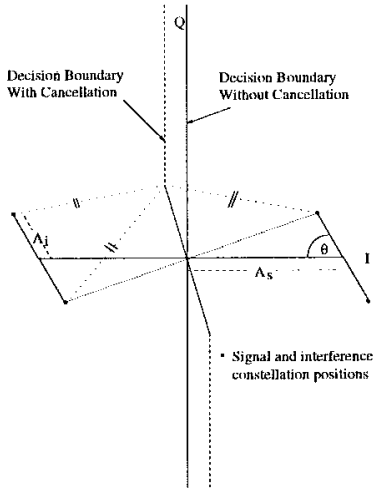

(a)

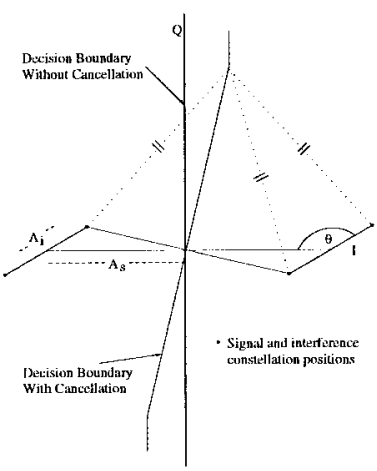

(c)

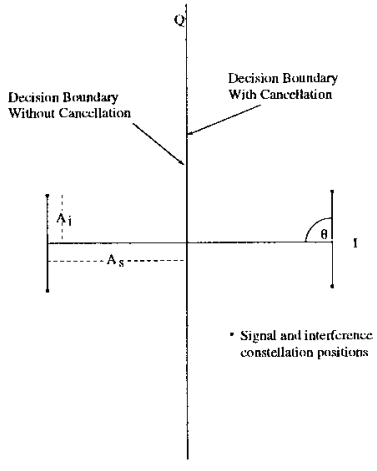

(b)

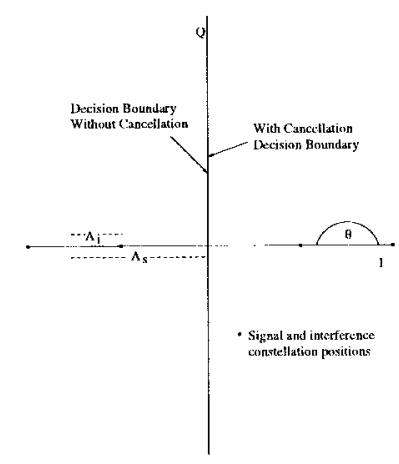

(d)
Fig. 11. Constellation diagrams of BPSK signal, with a phase noncoherent BPSK cochannel interferer at 8-dB SIR, before the addition of AWGN for various $\theta$ values showing the decision boundary with and without interference cancellation. (a) $\theta=\pi / 3$. (b) $\theta=\pi / 2$. (c) $\theta=5 \pi / 6$. (d) $\theta=\pi$ or 0 .

The analytical performance is given below for BPSK and QPSK. The results are confirmed by simulation and simulation results are also provided for the analytically less tractable square 16- and 64-QAM schemes.

1) Theoretical Performance of BPSK over Interfered Gaussian Channels with Interference Cancellation: It is assumed that the interfering signal can have any relative phase with respect to the desired signal. The effective angle between the two constellations in the baseband is given by $\theta$, where all values of $\theta$ are equally likely. The amplitude of the signal is given by $A_{s}$ and the amplitude of the interferer is given by $A_{i}$. The effect of corrupting the BPSK signal with a BPSK interferer generates an interfered received signal constellation, constituted by four phasors, as shown in Fig. 11. The figure includes the conventional decision boundaries for BPSK, as would be used in the absence of interference. Given the knowledge of the channel magnitude and phase as well as the type of the interfering phasor constellation, the cancellation of interference can be carried out by modifying the decision boundaries appropriately, in order to improve the BER performance. Specifically, we capitalize on this knowledge by adjusting the decision boundaries, such that they remain equidistant from the interfered constellation points. This results in a relatively simple geometric scenario in Fig. 11 for BPSK, where the equal distances are clearly marked, but becomes less intuitive for higher order modulation schemes. 
In addition, we note that in the uplink, i.e., at the BS, it would be possible to invoke so-called joint-detection schemes, which could jointly detect both the desired signal and the interference, rather than to cancel the interference, similarly to those in code-division multiple-access (CDMA) systems [27]. This solution is, however, less amenable to employment in the downlink, an issue, which is the subject of our future work. ${ }^{3}$

In order to clarify the above geometric constraint further, when using interference cancellation, the decision boundary is constituted by a locus of points equidistant from two out of the four constellation points of the combined BPSK signal plus superimposed BPSK interferer. These two interfered phasor points must not be associated with the same useful phasor, consequently, they are always chosen such that both of the possible useful phasor points are represented. To elaborate further, the equidistant criterion must be met with respect to those two specific phasor points which are at the minimum possible Euclidean distance from each other given the set of four phasors. In Fig. 11(b) and (d) the decision boundaries with cancellation are the same as those without. However, in Fig. 11(a) and (c) the decision boundaries are constituted by three linear sections. The break point between the adjacent boundary sections is where three constellation points are equidistant from the decision boundary, as suggested by the marked distances.

From the geometry of the figure it can be shown that the coordinates for the two decision boundary break points are given by

$$
\left(A_{s}-A_{i} \cos (\theta),\left(A_{s}-A_{i} \cos (\theta)\right) \cdot A_{i} \cos (\theta)\right)
$$

and

$$
\left(A_{s}+A_{i} \cos (\theta),\left(A_{i} \cos (\theta)-A_{s}\right) \cdot A_{i} \cos (\theta)\right) .
$$

The decision boundary for a BPSK signal with a BPSK interferer will be a linear function passing through these two points and vertical outside this range. However, the shape of the decision boundary will always depend on the actual value of $\theta$.

Taking the above three decision boundary sections of the interference-canceled, BPSK-interfered BPSK modem into account, it can be shown that the BER of the modem over a Gaussian channel can be calculated by

$$
P_{b}^{\prime \prime}\left(A_{s}, A_{i}\right)=\int_{\theta=0}^{\pi} \int_{y=-\infty}^{\infty} P_{e}(y) \frac{1}{\sqrt{2 \pi} \sigma} \exp \left(-y^{2} / 2 \sigma^{2}\right) d y d \theta
$$

where $P_{e}(y)$ takes the values shown in (13) at the bottom of this page, and $y$ is the quadrature component of the

\footnotetext{
${ }^{3}$ The comments of the anonymous reviewer concerning this issue are gratefully acknowledged.
}

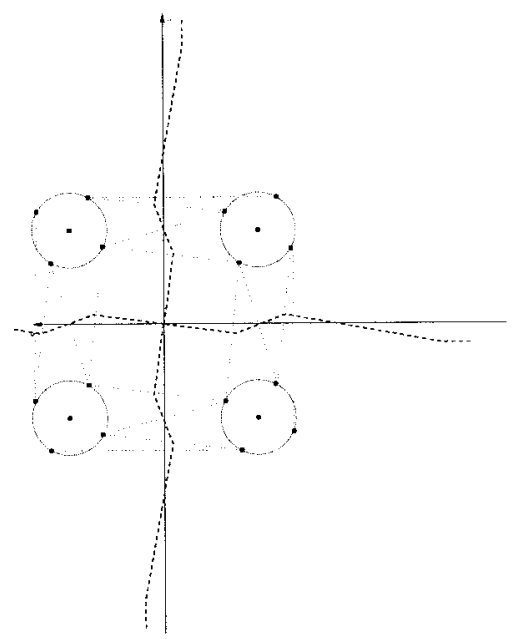

Fig. 12. QPSK decision boundaries with interference cancellation.

noise, while $W=-1$ if $\theta<\pi / 2$ or $W=+1$ if $\theta>\pi / 2$. The arguments of the Gaussian $Q($ ) function are based upon the distance of the constellation point, with $y$ added to the quadrature component, from the decision boundary. The solution to these equations was evaluated numerically, where $N$ was the noise power, such that $N=2 \sigma^{2}$ and the limits of the integration with respect to $y$ were approximated by $\mp 6 \sigma$. The translated decision boundaries take into account the effect of a single interferer and therefore attempt to compensate for the interference. The corresponding BER performance curves will be compared to simulated results in Fig. 13(a).

2) Theoretical Performance of QPSK over Interfered Gaussian Channels with Interference Cancellation: Expressing the performance of QPSK with a single QPSK interferer and interference cancellation is considerably more complex than with BPSK and a single BPSK interferer. In the case of BPSK the decision boundary with interference cancellation was defined over three regions and the symbol error rate was equal to the BER. However, for QPSK the corresponding decision boundary is defined for 28 regions in order to determine the BER. Therefore, a technique to determine the theoretical performance was exploited that did not require explicit evaluation of the decision boundaries. This was achieved by considering the QPSK phasor constellation, with a QPSK interferer similarly to Fig. 11, which results in a 16-point constellation, as portrayed in Fig. 12. The positions of all 16 points could be computed for all $\theta$ angles given the received signal and interference amplitudes, determined by the corresponding propagation channels. The decision boundaries were calculated by considering the halfway point along the shortest line between each of the constellation points and then bisecting that point with a perpendicular, as suggested by

$$
P_{e}(y)= \begin{cases}Q\left(A_{s}^{2} / N\right), & \text { if } y>\left(A_{s}-A_{i} \cos (\theta)\right) \cdot A_{i} \cos (\theta) \\ Q\left(\left(A_{s}-2 A_{i} \cos (\theta)\right)^{2} / N\right), & \text { if } y<\left(A_{i} \cos (\theta)-A_{s}\right) \cdot A_{i} \cos (\theta) \\ Q\left(\frac{\left(A_{s}-A_{i} \cos (\theta)-\left(\frac{\left(y+A_{i} \sin (\theta)\right) \cdot A_{i} \sin (\theta)}{W \cdot\left(A_{s}-A_{i} \cos (\theta)\right)}\right)^{2}\right)}{N}\right), & \text { otherwise }\end{cases}
$$




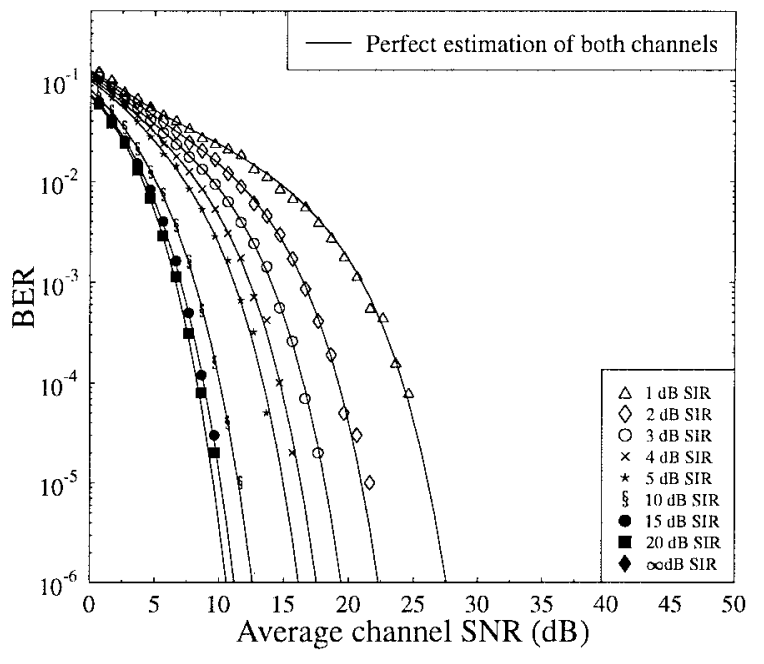

(a)

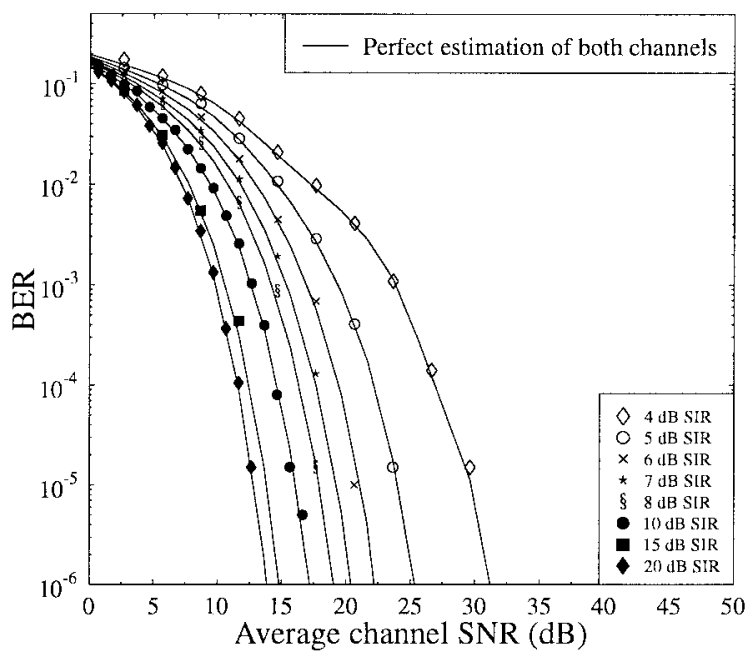

(b)

Fig. 13. Theoretical performance, with perfect magnitude and phase channel estimation (continuous line) and simulated performance with training sequence-based estimation (symbols), for BPSK and QPSK schemes over Gaussian channels with a single BPSK and QPSK interferer, at various SIR values, also received over a Gaussian channel using interference cancellation and $\alpha=0.35$. (a) BPSK. (b) QPSK

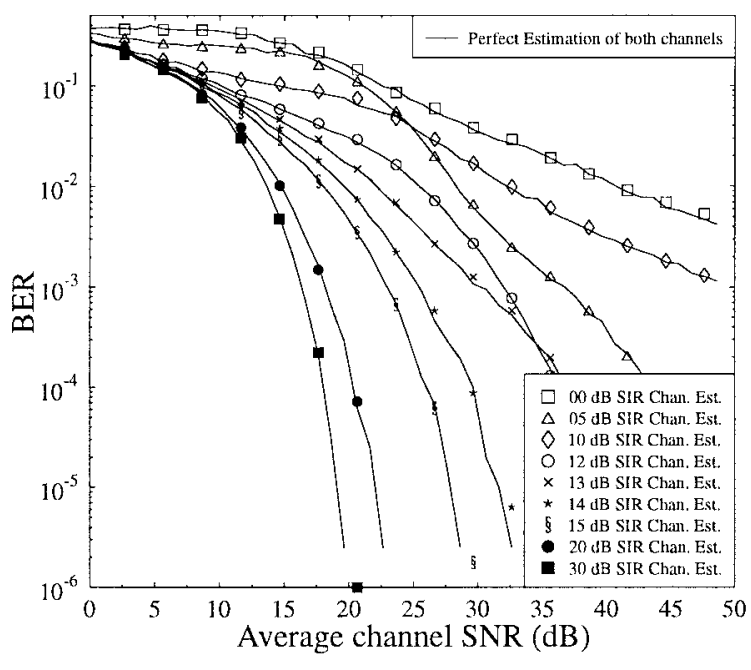

(a)

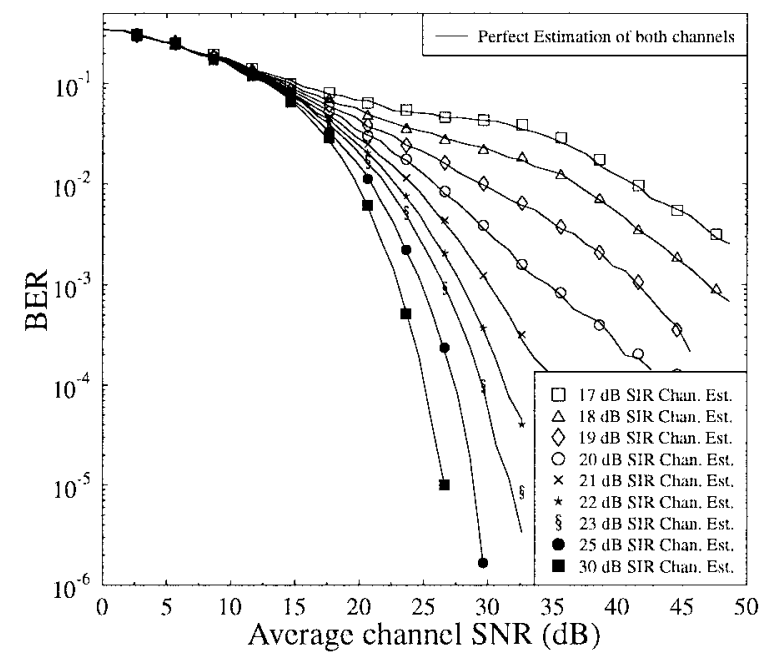

(b)

Fig. 14. Simulated performance, with perfect magnitude and phase channel estimation for both the interfered and the interfering channel (continuous line) and training sequence-based estimation for the interfering channel (symbols), for square 16- and 64-QAM schemes over Gaussian channels with a single square 16- and 64-QAM interferer, at various SIR values, also received over a Gaussian channel using interference cancellation and $\alpha=0.35$. (a) Square 16-QAM. (b) Square 64-QAM.

Fig. 12. This yielded $12 \times 16 / 2=384$ decision boundaries. The integral in (12) was then solved numerically, however, for QPSK this equation had to be solved in both the $x$ and $y$ directions order to compute the BER. The function $P_{e}(y)$ was evaluated on the basis of which boundary offered the least one-dimensional noise protection distance for a given $y$.

3) Simulated Performance over Interfered Gaussian Channels with Channel Estimation: Having derived the theoretical performance of the interference cancellation algorithm for BPSK and QPSK over interfered Gaussian channels, their performance may be compared with the simulated performance. When the simulation was conducted with perfect estimation of both the useful and interfering channel, there was extremely good correspondence between the simulated and numerical results. However, the more realistic scenario, where the a perfect estimation of the channel magnitude and phase was not assumed was also considered by simulation. The channel was estimated by employing two orthogonal sequences, 24 symbols long in the center of the TDD/TDMA burst, referred to as the mid-amble.

Fig. 13(a) shows a comparison between the numerical performance of BPSK with a single BPSK interferer, on the basis of perfect channel estimation (continuous line), and the simulated performance of BPSK with a single BPSK interferer, using the the mid-amble for estimation of the interfering channel magnitude and phase (symbols). Fig. 13(b) shows the same comparison for QPSK with a single QPSK interferer. In both cases it can be seen that there is little deterioration in performance resulting from using the mid-able for estimating the channel magnitude and phase. Fig. 14 shows the simulated 


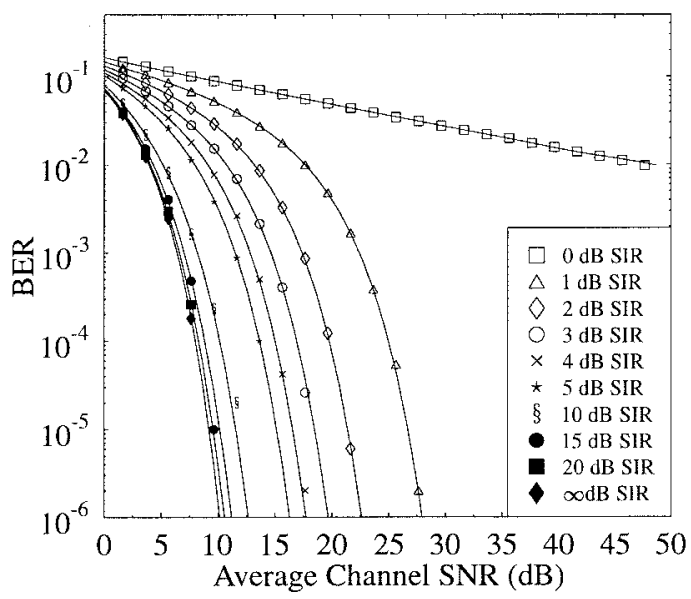

(a)

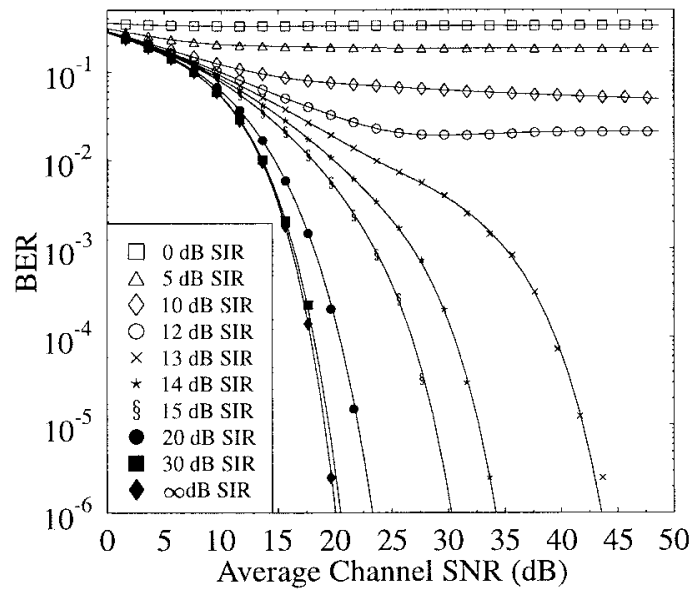

(c)

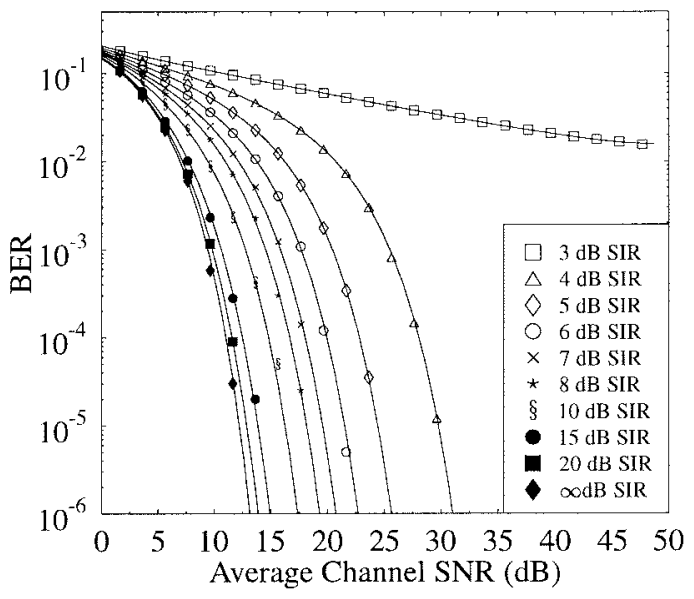

(b)

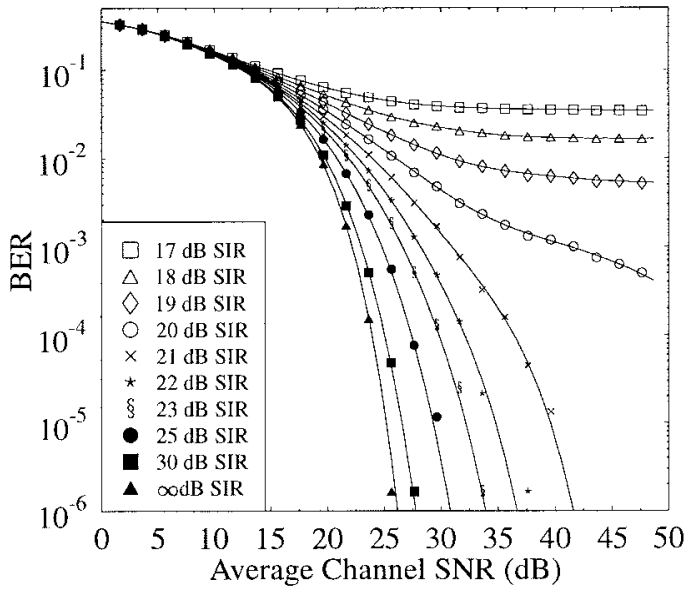

(d)

Fig. 15. Numerical and simulated performance of fixed-modulation schemes in Gaussian channels with the same modulation scheme interfering at various SIR values, when $\alpha=0.35$ and no interference cancellation. (a) BPSK. (b) QPSK. (c) Square 16-QAM. (d) Square 64-QAM.

performance of square 16- and 64-QAM with perfect channel estimation for both the interfered and the interfering channel (continuous line) and using the mid-amble to estimate the interfering channel (symbols). Again, it can be observed that the deterioration in performance resulting from using the midamble to estimate the channel is negligible.

Specifically, Fig. 13(a) shows the BPSK performance for SIR's greater than $0 \mathrm{~dB}$, and Fig. 13(b) portrays the QPSK performance for SIR's greater than $3 \mathrm{~dB}$, since the theory presented above is only valid for these respective ranges. For SIR's below these values there would be a residual BER even with infinite SNR. In Fig. 14(a) and (b) the same SIR values are used as in Fig. 15, because the results were generated by simulation and. therefore. there was no restriction upon the SIR that could be evaluated. It should be noted that in Fig. 14(a), the performance at 5- and 12-dB SIR, respectively, is better than at 10- and 13-dB SIR in the high average channel SNR range. This results from the complicated interaction of the $16^{2}=256$ combined signal and interference constellation points. When the maximum interference amplitude is higher than the distance between the square 16-QAM signal constel- lation points, the combined constellation points overlap for different $\theta$ values.

Having verified the model for interference cancellation with BPSK and QPSK, and shown that employing a mid-amble to estimate the phase and amplitude of the interfering channel, the performance of the fixed-modulation schemes over Gaussian channels is compared with and without interference cancellation. This is achieved by comparing Figs. 13 and 14 with Fig. 15. The effect of the interference cancellation upon the BPSK and QPSK modulation schemes is most significant at low SIR's and low SNR's. Fig. 13(a) compared with Fig. 15(a) reveals that at 1-, 2-, 3-, 4-, and 5-dB SIR, the SNR required for BER's in the region of $2-5 \%$ is reduced by as much as $3 \mathrm{~dB}$ by employing interference cancellation within BPSK modulation. Similar gains are recorded for QPSK by comparing Fig. 13(b) with Fig. 15(b). What is not shown is how the BER's are reduced at SIR's below $3 \mathrm{~dB}$ since, as stated above, the theoretical approach for evaluating the BER performance is not applicable at low SIR's.

However, for square 16- and 64-QAM the performance gains achieved using interference cancellation are more strik- 


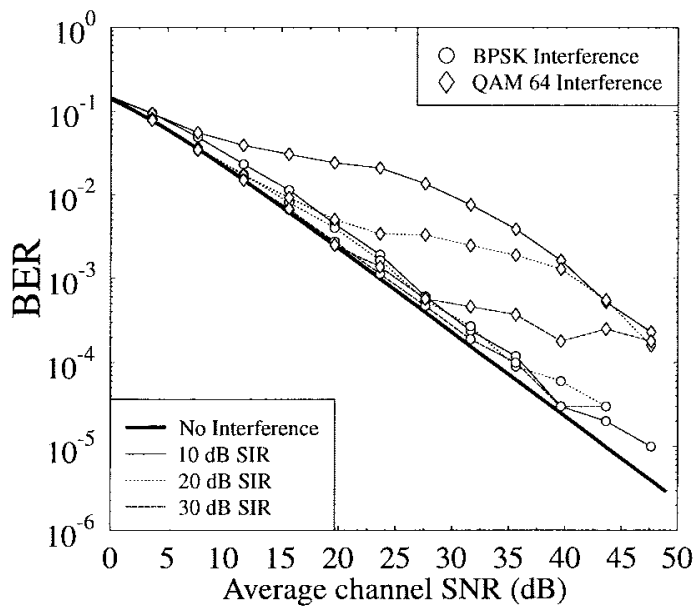

(a)

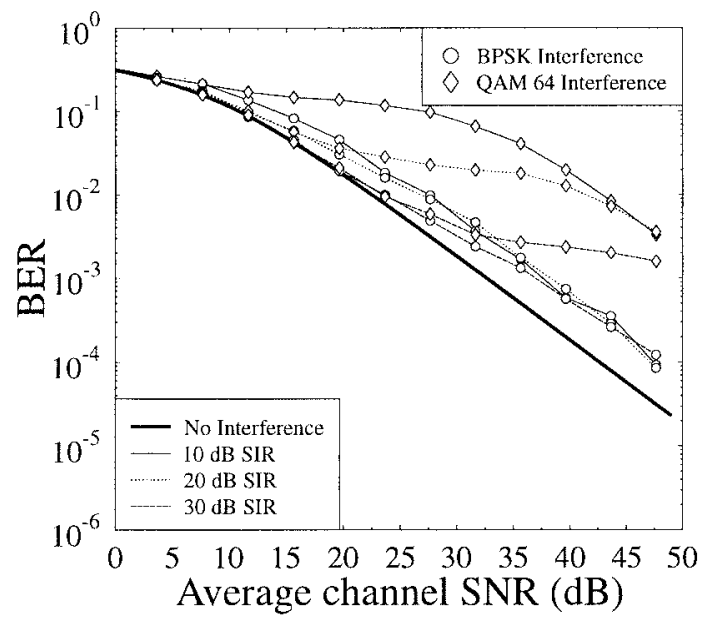

(c)

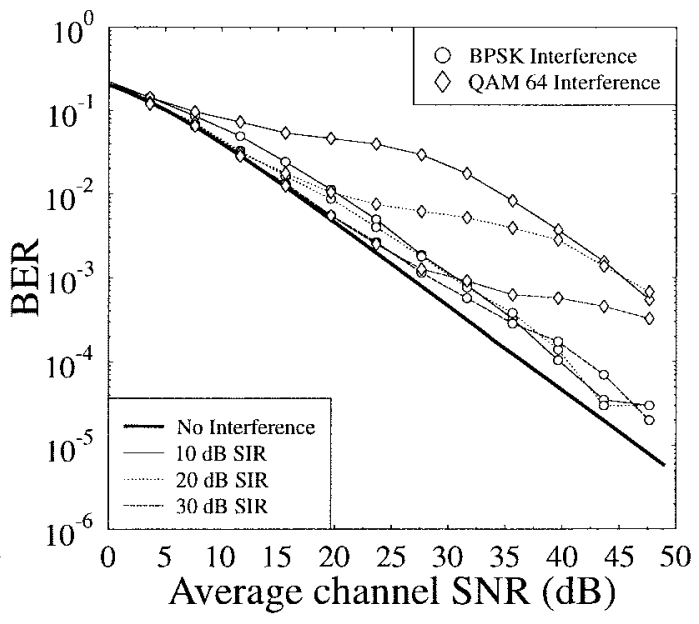

(b)

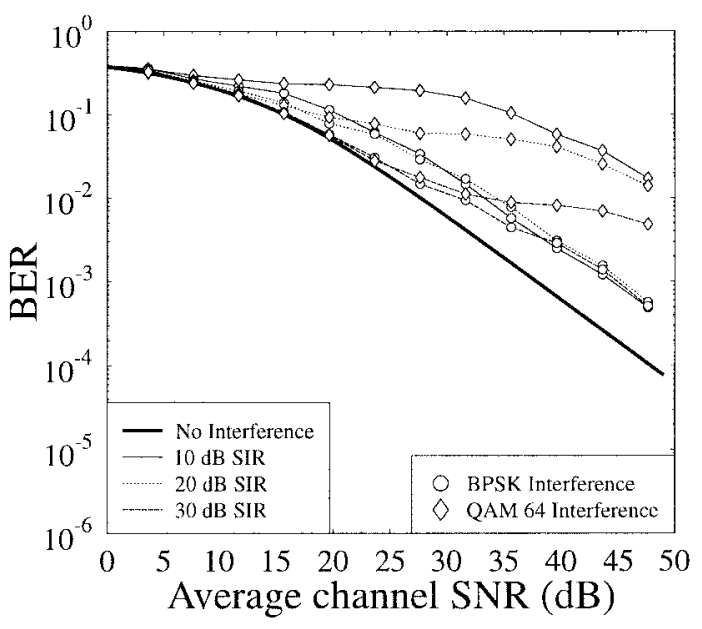

(d)

Fig. 16. Simulated performance of fixed-modulation schemes in slow Rayleigh channels with single interfering transmission at various SIR values also received via a Rayleigh channel using interference cancellation, estimated channel characteristics, and $\alpha=0.35$. (a) BPSK. (b) QPSK. (c) Square 16-QAM. (d) Square 64-QAM

ing. This is because the performance with interference cancellation was evaluated at SIR values where a residual BER existed without the cancellation. Consider Fig. 14(a) in comparison with Fig. 15(c), from which it can be identified that at $0-$ or 5-dB SIR, and at 30-dB SNR, the interference cancellation reduces the BER by an order of magnitude. At higher SNR's the improvement becomes as large as four orders of magnitude. In a realistic communications system the channel and network conditions are unlikely to result in a Gaussian line-of-sight scenario where the signal and interferer produce 0- or 5-dB SIR and 30-dB SNR, as implied by these Gaussian channel experiments. However, over fading channels with independent signal and interfering paths such SNR and SIR values could occur temporarily. Therefore, the performance is now evaluated assuming that both the signal and interferer are Rayleigh fading independently.

4) Interference Cancellation Performance over Rayleigh Channels Using Channel Estimation: The performance of each of the fixed modulation schemes is evaluated with either a single BPSK or single square 64-QAM interferer by simulation, when employing interference cancellation. This will reveal the performance of interference cancellation for each scheme in a Rayleigh fading channel and show, how it is affected by the least and most complex phase noncoherent time-synchronous interferer. The performance was evaluated by simulation and using the mid-amble to estimate the interfering channel's magnitude and phase. Both the signal and interferer were assumed to be constant over a time slot and $1 \times 10^{5}$ symbols were simulated for every SNR and SIR value. The results with interference cancellation are shown in Fig. 16, while the corresponding benchmark curves without cancellation are portrayed in Fig. 17. Their comparison reveals the following findings.

- Logically, the use of interference cancellation never results in the BER being lower than it would have been had there been no interference.

- At high SNR there is a margin between the performance obtained when there is no interference and the performance achieved when a single interferer is canceled. This gap increases, as the useful signal constellation's com- 
TABLE IX

Ber Reduction Due to Interference Cancellation with Fixed Modulation Schemes Transmitted Through a Slow Rayleigh Fading Channel with a Single Independent Slow Rayleigh Fading Interferer, Using the Mid-Amble to Estimate the Interfering Channel and Perfect Estimates for the Wanted Channel

\begin{tabular}{c|c|c|c|c|c|c|c}
\hline & SNR & \multicolumn{3}{|c|}{ BPSK Interferer } & \multicolumn{3}{c}{ QAM64 Interferer } \\
\cline { 3 - 8 } & $(\mathrm{dB})$ & $10 \mathrm{~dB}$ SIR & $20 \mathrm{~dB}$ SIR & $30 \mathrm{~dB}$ SIR & $10 \mathrm{~dB}$ SIR & $20 \mathrm{~dB}$ SIR & $30 \mathrm{~dB}$ SIR \\
\hline BPSK & 20 & $1.8 \times 10^{-2}$ & $1.0 \times 10^{0}$ & $1.0 \times 10^{0}$ & $1.0 \times 10^{0}$ & $1.0 \times 10^{0}$ & $1.0 \times 10^{0}$ \\
QPSK & 20 & $2.5 \times 10^{-2}$ & $1.0 \times 10^{0}$ & $1.0 \times 10^{0}$ & $1.0 \times 10^{0}$ & $1.0 \times 10^{0}$ & $1.0 \times 10^{0}$ \\
QAM16 & 20 & $2.8 \times 10^{-2}$ & $1.0 \times 10^{0}$ & $1.0 \times 10^{0}$ & $1.0 \times 10^{0}$ & $1.0 \times 10^{0}$ & $1.0 \times 10^{0}$ \\
QAM64 & 20 & $5.5 \times 10^{-1}$ & $1.0 \times 10^{0}$ & $1.0 \times 10^{0}$ & $1.0 \times 10^{0}$ & $1.0 \times 10^{0}$ & $1.0 \times 10^{0}$ \\
\hline BPSK & 30 & $1.5 \times 10^{-3}$ & $1.3 \times 10^{-1}$ & $1.0 \times 10^{0}$ & $4.1 \times 10^{-1}$ & $1.0 \times 10^{0}$ & $1.0 \times 10^{0}$ \\
QPSK & 30 & $2.5 \times 10^{-3}$ & $3.8 \times 10^{-1}$ & $1.0 \times 10^{0}$ & $5.0 \times 10^{-1}$ & $1.0 \times 10^{0}$ & $1.0 \times 10^{0}$ \\
QAM16 & 30 & $2.7 \times 10^{-2}$ & $3.4 \times 10^{-1}$ & $1.0 \times 10^{0}$ & $5.5 \times 10^{-1}$ & $1.0 \times 10^{0}$ & $1.0 \times 10^{0}$ \\
QAM64 & 30 & $1.8 \times 10^{-1}$ & $4.0 \times 10^{-1}$ & $1.0 \times 10^{0}$ & $9.0 \times 10^{-1}$ & $1.0 \times 10^{0}$ & $1.0 \times 10^{0}$ \\
\hline BPSK & 40 & $1.4 \times 10^{-4}$ & $2.5 \times 10^{-2}$ & $1.3 \times 10^{-1}$ & $6.8 \times 10^{-2}$ & $4.6 \times 10^{-2}$ & $7.8 \times 10^{-1}$ \\
QPSK & 40 & $6.8 \times 10^{-4}$ & $4.0 \times 10^{-2}$ & $3.4 \times 10^{-1}$ & $7.8 \times 10^{-2}$ & $5.1 \times 10^{-1}$ & $1.0 \times 10^{0}$ \\
QAM16 & 40 & $3.6 \times 10^{-3}$ & $3.9 \times 10^{-2}$ & $3.0 \times 10^{-1}$ & $1.7 \times 10^{-1}$ & $6.5 \times 10^{-1}$ & $1.0 \times 10^{0}$ \\
QAM64 & 40 & $1.1 \times 10^{-2}$ & $4.6 \times 10^{-2}$ & $3.5 \times 10^{-1}$ & $2.5 \times 10^{-1}$ & $8.0 \times 10^{-1}$ & $1.0 \times 10^{0}$ \\
\hline
\end{tabular}

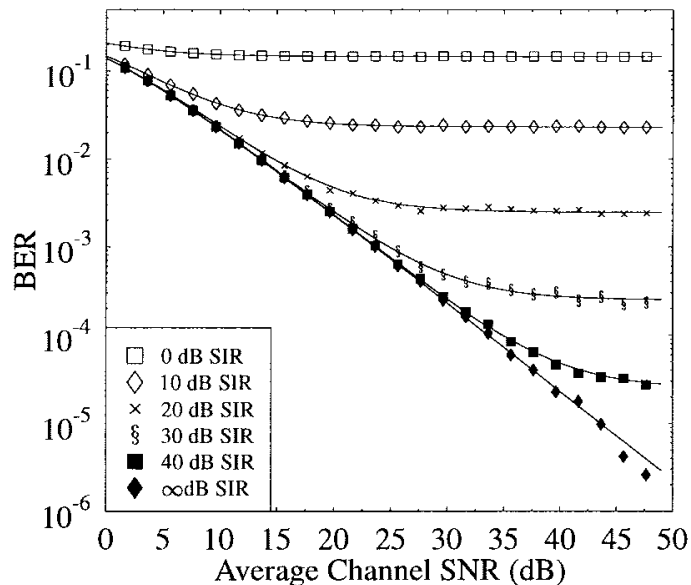

(a)

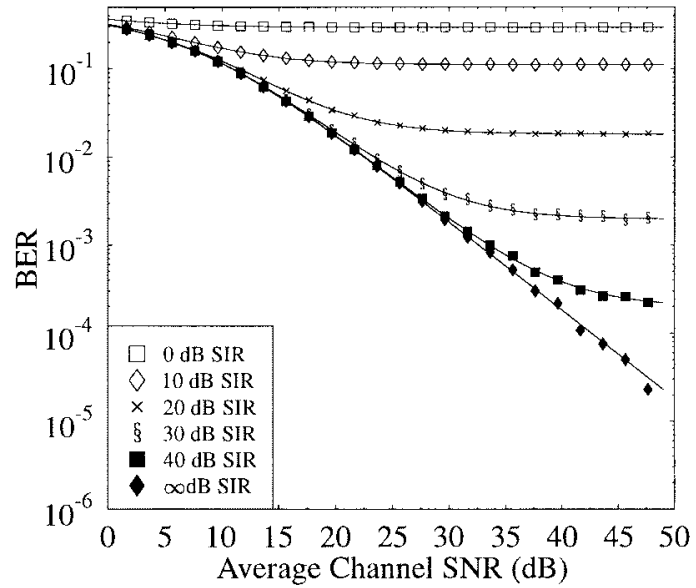

(c)

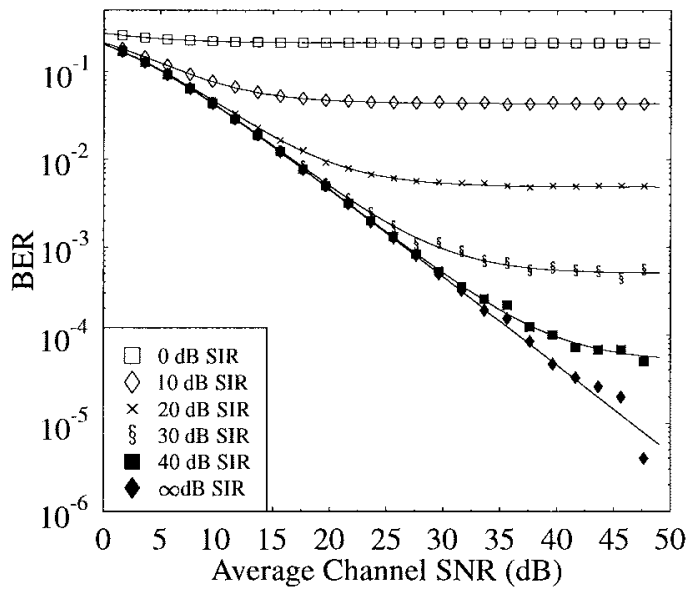

(b)

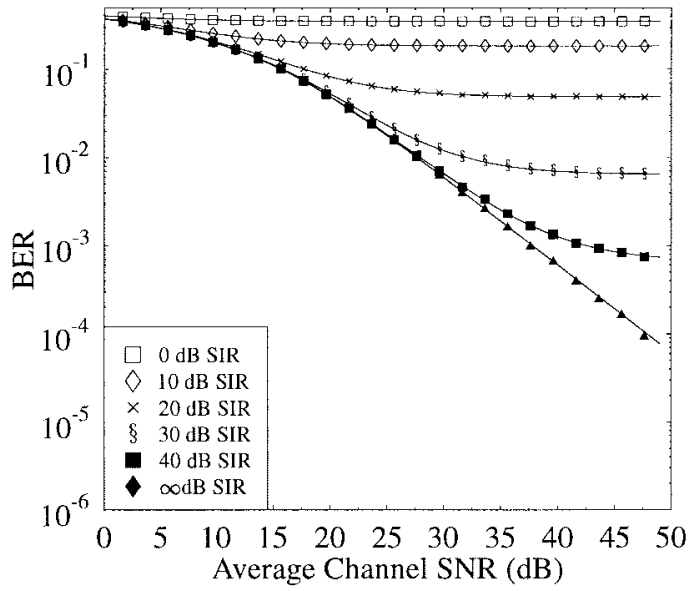

(d)

Fig. 17. Simulated performance of fixed-modulation schemes in Rayleigh channels without interference cancellation at various SIR values, where the interfering modulation scheme was the same as the useful signal and received over an independent Rayleigh channel. $\alpha=0.35$ was used. (a) BPSK (b) QPSK. (c) Square 16-QAM. (d) Square 64-QAM

plexity increases. The latter phenomenon can be explained by considering Figs. 11 and 12, which show the constellation of possible received phasors, if a single BPSK interferer is superimposed upon a BPSK transmission or a QPSK interferer corrupts a QPSK user, respectively.
Generally, the cancellation decision boundary results in superior BER performance in comparison to the original decision boundary without cancellation. However, as discussed before, the boundary translation does not fully mitigate the effects of interference. This is because as 
the combined signal and interferer constellation becomes more complex, the distance of the constellation points is reduced in comparison to the original uninterfered constellation.

- At low SNR's the BER performance is limited by the noise, irrespective of the SIR. As the SNR increases, it becomes constrained by the level of SIR and the interfering modulation scheme, where square 64-QAM is clearly more detrimental. Lastly, at high SNR's, the BER performance is limited only by the interfering modulation scheme. The latter observations are best explained by comparing the parts in Fig. 16 with the corresponding results in Fig. 17. It is the case for all signaling schemes with both of the interfering schemes, but more clearly seen with the square 64-QAM interferer, shown in Fig. 16 that the BER curves with interference and cancellation initially decay with increased SNR, then experience a range of SNR's where the BER performance levels out, and then the BER reduces again with SNR. However, by considering Fig. 17 it can be observed that the SNR regions where the BER performance levels out in Fig. 16 correspond to the corner SNR's, where the BER residual was experienced without interference cancellation for the given SIR's. Clearly, in this flat region the interference cancellation is unable to function, because the noise is corrupting the combined constellation of signal and interferer. At SNR's beyond the flat region the interference cancellation begins to have a significant positive effect. The difference between the length of the flat BER region with a BPSK compared to a square 64-QAM interferer is due to the reduced noise tolerance of the combined signal and interference constellation, resulting from any of the signaling schemes with a single BPSK interferer compared with single square 64-QAM interferer.

Table IX summarizes the advantage of employing interference cancellation, when comparing Fig. 16 with Fig. 17. The interference cancellation achieves the maximum performance, when the signal and interference constellations are least complicated. That is, interference cancellation is most useful, when employed in conjunction with low-order modulation schemes. The BER is reduced by interference cancellation the most, when the average SIR is low, but the SNR is high. That is, when the structure of the combined signal and interference constellation is most clearly defined.

\section{Adaptive Schemes}

Having studied fixed-modulation schemes assisted by interference cancellation, employing interference cancellation in conjunction with adaptive modulation is considered. These experiments are based upon the assumptions used in our previous deliberations; however, it is additionally assumed that there is an equal probability of a "no transmission," BPSK, QPSK, square 16- or 64-QAM symbol interfering with the data transmission, and that the modulation schemes used in both the signal and interfering channels are known at the receiver. The transmission of this control information was discussed in [12] by the authors, where a nonuniform five-phasor PSK constellation was introduced for their signaling.

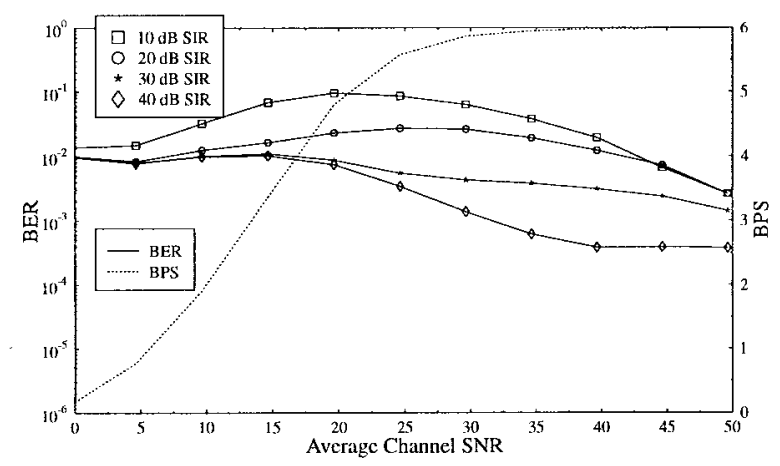

Fig. 18. Downlink BER over a slow Rayleigh fading channel with various SIR levels at the MS and no interference at the BS, the original optimized adaptive speech switching levels and interference cancellation with perfect estimation and mid-amble estimation of the signal and interference channels, respectively, where $\alpha=0.35$.

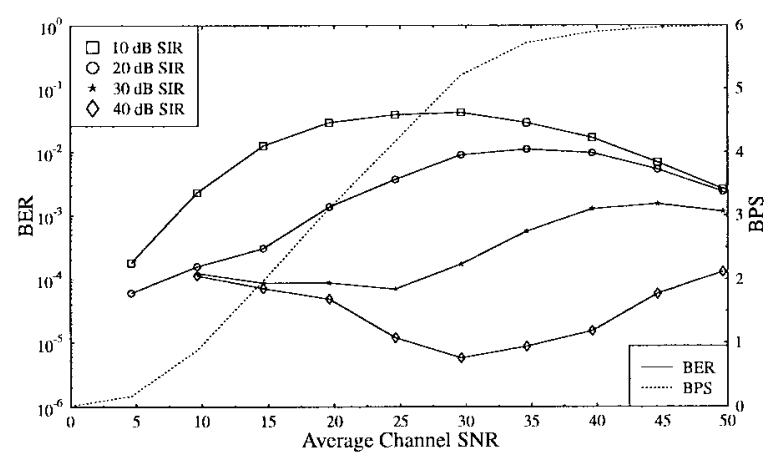

Fig. 19. Downlink BER over a slow Rayleigh fading channel with various SIR levels at the MS and no interference at the BS, the original optimized adaptive computer data switching levels and interference cancellation with perfect estimation and mid-amble estimation of the signal and interference channels, respectively, where $\alpha=0.35$.

Figs. 18 and 19 show the BER and BPS performance of the optimized mean BER, speech, and computer data scheme with switching levels given in Table I, i.e., with switching levels ignoring the effects of interference, over slow Rayleigh fading channels with various levels of CCI from a single interferer, when interference cancellation is employed. The results were generated with perfect estimation of the wanted channel and exploitation of the mid-amble to estimate the interfering channel. These results may be compared with the same performance in the absence of interference cancellation shown in Figs. 6 and 7. For both the speech and computer data schemes at 10-, 20-, 30-, and 40-dB SIR interference cancellation improves the BER performance. It is clear that the performance improves most at high average channel SNR values, and this is what would be expected, bearing in mind the results from Section IV-C. However, the speech system still fails to achieve the BER target of $1 \times 10^{-2}$ for some average channel SNR's at 10- and 20-dB SIR. As seen in the figure, in the case of the computer data system the BER performance curve also fails to achieve the target BER of $1 \times$ $10^{-4}$, when interference cancellation is employed if the SIR is lower than $40 \mathrm{~dB}$.

As seen in the captions, the downlink results shown in Figs. 18 and 19 are based upon estimating the expected 
TABLE X

Manually Determined Switching Levels, in Decibels, FOR AdAPtive Schemes over RAYleigh FAding CHANNElS EXPERIENCING COCHANNEL INTERFERENCE AND EMPLOYING INTERFERENCE Cancellation, Where the Performance Is Shown in Figs. 20 and 21

\begin{tabular}{c|c|c|c|c|c|c|c|c}
\hline & \multicolumn{4}{|c|}{ Speech } & \multicolumn{4}{c}{ Computer Data } \\
\hline SIR (dB) & $l_{1}$ & $l_{2}$ & $l_{3}$ & $l_{4}$ & $l_{1}$ & $l_{2}$ & $l_{3}$ & $l_{4}$ \\
\hline 10 & 4 & 10 & 27 & 35 & \multicolumn{5}{c|}{ Unused } \\
\cline { 5 - 9 } 20 & 3 & 6 & 12 & 30 & 14 & 30 & 38 & 60 \\
30 & 3 & 6 & 12 & 18 & 8 & 11 & 17 & 60 \\
40 & 3 & 6 & 12 & 18 & 8 & 11 & 17 & 25 \\
\hline
\end{tabular}

downlink channel quality on the basis of an interferencefree uplink. In other words, the uplink transmission is used as a measure of the channel quality, in order to estimate the conditions for the next downlink transmission and to decide which modulation scheme should be employed - in the absence of interference. Although this is an unlikely scenario in a cellular environment, here this assumption is used to allow comparison with the earlier figures. Furthermore, when interference cancellation is employed, this situation is much more realistic, since both the signal and interferer channels are estimated by separate orthogonal mid-ambles, as explained in Section IV-A, minimizing the effects of interference upon the channel estimation.

In order to achieve the target BER's of $1 \times 10^{-2}$ and $1 \times 10^{-4}$ in the presence of interference, simply using the switching levels from Table I and employing interference cancellation is clearly not sufficient. A feasible approach to achieving the target BER's for both schemes across the desired range of average channel SNR's would be to adaptively adjust the switching levels for different average channel SNR's. In other words, this would imply that the switching levels would be varied depending upon the prevailing average channel SNR conditions, potentially requiring increased switching thresholds for maintaining the target BER at low SNR's. We note, however, that there would be a concomitant BPS performance penalty. However, the drawback of this approach is that it would involve accurate estimation of the average signal and interference levels. Therefore, we opted for using the same switching levels for all average channel SNR's, but varying them on the basis of the average interference level. The estimation of the prevalant SIR's is left for further study, following the approach of Andersin [20].

In order to obtain optimum switching levels for Rayleigh fading channels in the absence of noise and with CCI, without employing interference cancellation, Powell optimization [18] has been used, as in Section III-B. This has been possible, because a numerical solution to the BER and PBS performance of adaptive modulation was derived for these cases and therefore iterative optimization has been feasible. However, a full numerical solution for adaptive modulation, with an independent cochannel interferer and cancellation, has not been found. Therefore, the switching levels are derived by an iterative manual technique.

The interference cancellation was simulated with the perfect magnitude and phase estimations of the wanted signal channel and mid-amble based results for the interfering channel. The proposed switching levels are given in Table $\mathrm{X}$ and the

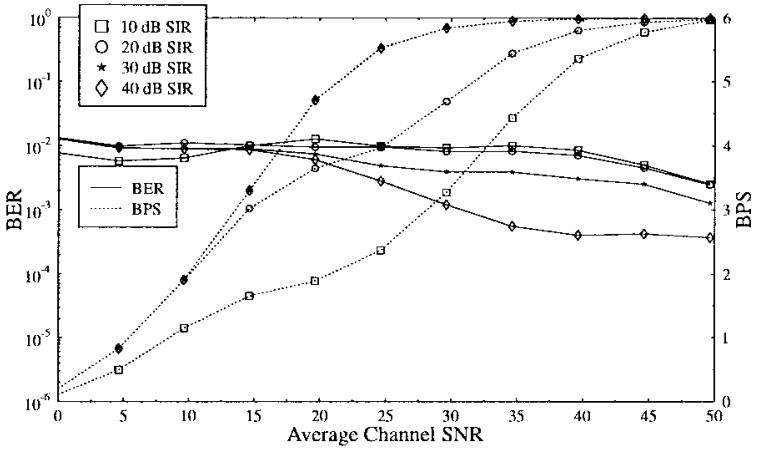

Fig. 20. Downlink BER over a slow Rayleigh fading channel with various SIR levels at the MS and no interference at the BS, the manually adjusted for interference cancellation adaptive speech switching levels and interference cancellation with perfect estimation and mid-amble estimation of the signal and interference channels, respectively, where $\alpha=0.35$.

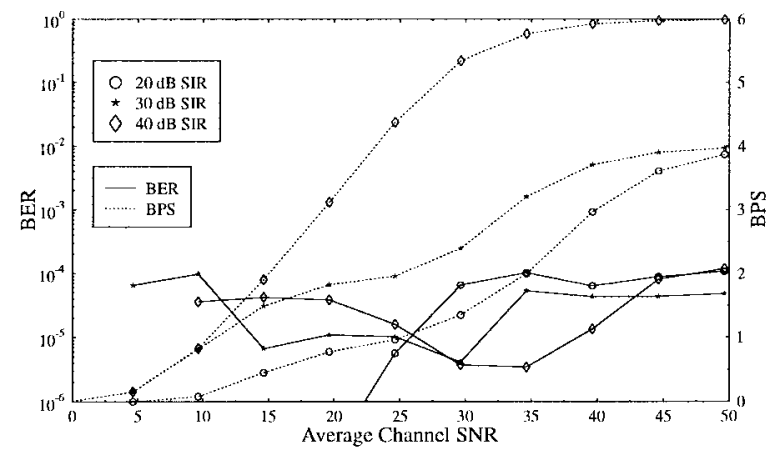

Fig. 21. Downlink BER over a slow Rayleigh fading channel with various SIR levels at the MS and no interference at the BS, the manually adjusted for interference cancellation adaptive computer data switching levels and interference cancellation with perfect estimation and mid-amble estimation of the signal and interference channels, respectively, where $\alpha=0.35$.

associated performance curves are shown in Figs. 20 and 21. The switching levels of Table $\mathrm{X}$ may be compared with the reoptimized switching levels in the presence of interference, but without interference cancellation that were shown in Tables VI and VII. This comparison, for the speech system, shows that only the $l_{4}$ values are changed significantly. In the case of 10$\mathrm{dB}$ SIR, $l_{4}$ is increased to $35 \mathrm{~dB}$ and at $20-\mathrm{dB}$ SIR it is reduced to $30 \mathrm{~dB}$. These changes in switching levels, for the speech system, used in conjunction with interference cancellation allow the target BER of $1 \times 10^{-2}$ to be maintained over the range of average channel SNR's from 0 to $50 \mathrm{~dB}$ and SIR's of $10,20,30$, or $40 \mathrm{~dB}$, which is shown in Fig. 20. This was not the case when the switching levels from Table VI were used, without interference cancellation, in the presence of a single phase-noncoherent time-synchronous Rayleigh fading interferer at SIR's of 10 or $20 \mathrm{~dB}$, as was shown in Fig. 8. Therefore, making the modifications to $l_{4}$ mentioned above, with respect to the reoptimized switching levels and invoking interference cancellation implies that adaptive modulation may be used for transmission with an average BER below $1 \times 10^{-2}$ over a Rayleigh fading channel for average channel SNR's from 0 to $50 \mathrm{~dB}$ and for SIR's of $10 \mathrm{~dB}$ or greater, when considering the worst case scenario of a single interfering adaptive modem. 


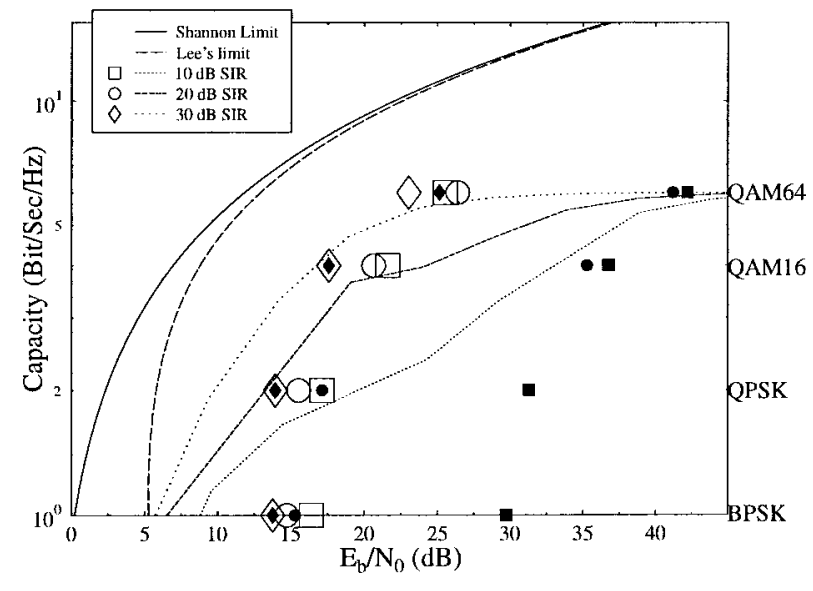

Fig. 22. Capacity limit and upper-bound performance of fixed and adaptive modulation schemes in Rayleigh fading channels for specific BER of $1 \times$ $10^{-2}$. Signaling and pilot overheads were neglected, assuming perfect filtering and single-sided bandwidth with $\alpha=0$. A single Rayleigh fading cochannel interferer at 10-, 20-, and 30-dB SIR was used for adaptive schemes, shown with lines, and 10-, 20-, and 30-dB SIR's were employed for the fixed schemes shown with markers. Interference cancellation was invoked, where "large hollow" markers represent BPSK interference and "small bold" markers correspond to square 64-QAM interference.

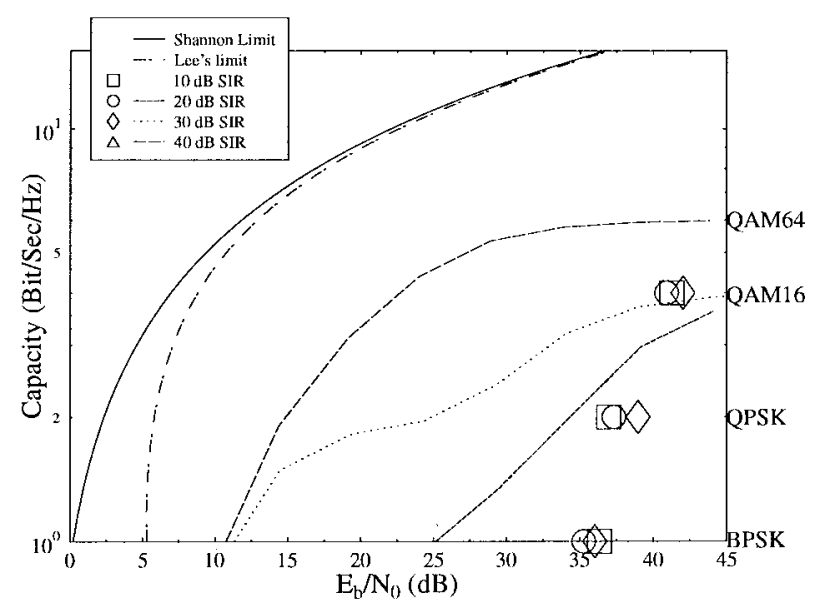

Fig. 23. Capacity limit and upper-bound performance of fixed and adaptive modulation schemes in Rayleigh fading channels for specific BER of $1 \times$ $10^{-4}$. Signaling and pilot overheads were neglected, assuming perfect filtering and single-sided bandwidth with $\alpha=0$. A single Rayleigh fading cochannel interferer at 10-, 20-, and 30-dB SIR was used for adaptive schemes, shown with lines, and 10-, 20-, and 30-dB SIR's were employed for the fixed schemes shown with markers. Interference cancellation was invoked, where "large hollow" markers represent BPSK interference. There are no "small bold" markers in this figure to represent square 64-QAM interference, since these values fell outside the plotted range.

Now the computer data system with a target BER of 1 $\times 10^{-4}$ is considered. First, upon comparing Table $\mathrm{X}$ with Table VII, it can be observed that the values of most of the switching levels are reduced, except at the combination of low SNR's and high SIR's, where the noise is dominant and the interference cancellation yields no significant benefits. However, Fig. 21 shows that despite this, the BER performance compared with Fig. 9 is improved, in terms of the range of SNR and SIR, for which the target BER can be achieved. The potential benefits associated with the switching levels being varied depending upon the average channel SNR conditions can be seen in Figs. 21 and 9, when the SIR is low. It can be seen, for example, in Fig. 21 that with 20-dB SIR and 22-dB average channel SNR, the BER performance is very much below the target BER of $1 \times 10^{-4}$. Under these channel conditions lower switching levels would be desirable, since then additional BPS capacity would be yielded. However, the BER is considerably closer to the target level, as the SNR increases, and since the same switching levels are used for all average channel SNR's, the switching levels cannot be reduced. For this reason, switching the modulation scheme on the basis of the corrupted bits detected by a channel codec, as proposed in [1]-[3] may be preferable, although a range of other associated issues must be considered, when invoking this principle. In summary, it should be noted that the target BER is achieved for all average channel SNR's and SIR's that are shown in Fig. 21. Furthermore, that the proposed manual switching levels, with interference cancellation, for high SIR's correspond closely to the Powell-optimized [18] switching levels given in Table I, for both speech and computer data systems. Before concluding, let us now consider the achieved channel capacity of our adaptive modem in the next section.

\section{Channel CAPACITY}

The above discussion has focused upon the BER and BPS performance of various modems, but it is also of considerable interest to compare the relative channel capacities of the fixed and adaptive schemes by also considering the effects of interference and interference cancellation. This type of comparison is not as pertinent as the radio capacity or area spectral efficiency [23] technique used for example by Webb, although it dispenses with the need for a microcellular design propagation system. Figs. 22 and 23 show the capacity that may be achieved for a given $E_{b} / N_{0}$, using fixed and adaptive modulation schemes with target BER's of $1 \times 10^{-2}$ and 1 $\times 10^{-4}$, over a slow Rayleigh fading channel, assuming a single interferer at various SIR's and the use of interference cancellation. The fixed scheme's performance was derived from Fig. 16. The rolloff factor $\alpha$ is set to zero for normalization purposes, and the "small bold" markers represent the performance of fixed schemes, when the interfering signal is square 64-QAM, while the "large hollow" markers represent the performance for fixed schemes, when the interfering symbol is BPSK. The adaptive results are derived from Figs. 20 and 21. Considering Fig. 22, it can be seen that the adaptive performance at 10-dB SIR is closer to Shannon's or Lee's [17] channel capacity limit than the fixed 1-, 2-, and 4-BPS schemes with the same level of interference, when they are corrupted by square 64-QAM and interference cancellation is invoked. This is also the case, when comparing the adaptive scheme with the fixed 1-BPS scheme and a single BPSK interferer.

However, the other fixed schemes outperform the adaptive modem at 10-dB SIR. At 30-dB SIR, however, only the 6-BPS fixed scheme can outperform the adaptive scheme, irrespective of the type of interferer. Considering Fig. 23, which shows the adaptive data system's performance at $1 \times 10^{-4} \mathrm{BER}$, it can be seen that the $10-\mathrm{dB}$ SIR adaptive scheme is not represented, and neither are the fixed schemes with 64-QAM 
interferers. This is, because the maximum average channel SNR's of Figs. 16, 20, and 21 are insufficient to determine the $E_{b} / N_{0}$, at which a BER of $1 \times 10^{-4}$ is achieved. In some cases, the required $E_{b} / N_{0}$ could be very high, nonetheless, Fig. 23 shows that at $30-\mathrm{dB}$ SIR and above the adaptive scheme is generally more efficient than the fixed schemes with any interferer and at $20 \mathrm{~dB}$ it is more efficient than the fixed schemes with square 64-QAM interferes.

Figs. 22 and 23 give a summary of the channel capacity performance of the fixed and adaptive schemes, with interference and interference cancellation. However, it is important to note that they neglect the exact BER's encountered other than stating that a specific average target has been achieved. It is important to note, finally, that the higher BER speech system exhibited a substantially higher channel capacity than the lower rate data system, which is a consequence of allowing a more frequent employment of the higher order constellations and tolerating the associated higher BER.

\section{CONCLUSION}

In this paper our discussions were centered around the optimum choice of adaptive modem switching levels required to maintain target BER's of 1 and $0.01 \%$, respectively, under interfered conditions. It was shown in Fig. 1 that adjacent and cochannel interference have approximately the same effect upon the BER, for a given SIR, over Rayleigh fading channels. The effects of cochannel interference for fixed-modulation schemes in Gaussian channels were given by numerical integration and simulation in Fig. 15.

Cochannel interference has potentially a double negative effect upon adaptive modulation. Figs. 4 and 5 showed the effect of interference upon the BER of adaptive modems in the artificial scenario where the interference only corrupts the channel estimate. This problem can be neglected in a system that invokes interference cancellation. The effect of the interference upon the received symbols was shown in Figs. 6 and 7, where there was clearly a significant degradation of BER performance. However, this problem was addressed to a certain extent with reoptimized switching levels, given in Tables VI and VII, yielding BER performance curves shown in Figs. 8 and 9.

Interference cancellation offered a more complete solution, especially when combined with manual adjustment of the adaptive switching levels. Initially, however, the effect of interference cancellation was characterized for fixed modulation, yielding the gains given in Table IX. The manual adjustment and interference cancellation results for adaptive modulation yielded a guaranteed BER of $1 \times 10^{-2}$ and $1 \times 10^{-4}$ for the speech and computer data schemes for a range of values, shown in Figs. 20 and 21.

In conclusion, adaptive modulation is best suited to benign, low-interference indoor environments. This is because TDD is the most appropriate scheme to estimate the channel conditions, and unequalized indoors TDD requires sufficiently low propagation delays. Adaptive modulation is also most suitable for low indoors pedestrian velocities. In such an indoors environment, cochannel interference would be mitigated by walls and ceilings in the building. However, there is scope for interference to be produced on a short-term basis, when a combination of doors are opened, internal partitions are moved inside the building, or a vehicle passes the building, reflecting some potentially interfering signal back into the property. Such examples of interference are likely to result in single interferes, a scenario which is amenable to interference cancellation. The results shown in Figs. 22 and 23 demonstrate that adaptive modulation can achieve significant capacity gains compared with fixed-modulation schemes in such situations. The benefits of adaptive modulation are greater than shown in Figs. 22 and 23 because the instantaneous BER of the adaptive scheme is often significantly lower than the target. Furthermore, the comparison with the fixed-modulation schemes was conducted assuming that the most appropriate fixed scheme for the prevalent average channel conditions was employed. Clearly, by definition, the mobile will move from one position to another, this will have an effect upon the average channel conditions. Therefore, some dynamic change in the fixed modulation scheme is implicitly assumed during the comparison between fixed and adaptive schemes. Our future work in this area is focused on invoking BER estimates, rather than signal estimates, for assessing the channel quality.

\section{REFERENCES}

[1] R. Steele and W. T. Webb, "Variable rate QAM for data transmission over Rayleigh fading channels," in Wireless '91, IEEE, Calgary, Alta., Canada, 1991, pp. 1-14.

[2] W. Webb and R. Steele, "Variable rate QAM for mobile radio," IEEE Trans. Commun., vol. 43, pp. 2223-2230, July 1995.

[3] W. T. Webb and L. Hanzo, Modern Quadrature Amplitude Modulation: Principles and Applications for Fixed and Wireless Channels. New York: IEEE Press-Pentech Press, 1994, p. 557.

[4] S. Sampei, S. Komaki, and N. Morinaga, "Adaptive modulation/TDMA scheme for large capacity personal multi-media communication systems," IEICE Trans. Commun., vol. 77. no. 9, pp. 1096-1103, 1994.

[5] S. Otsuki, S. Sampei, and N. Morinaga, "Square-QAM adaptive modulation TDMA/TDD systems using modulation level estimation with Walsh function," Electron. Lett., pp. 169-171, Nov. 1995.

[6] S.-G. Chua and A. Goldsmith, "Variable-rate variable-power mqam for fading channels," in 46th Vehicular Technology Conf., IEEE, 1996, pp. 815-819.

[7] M.-S. Alouini and A. Goldsmith, "Area spectral efficiency of cellular mobile radio systems," in Proc. IEEE VTC'97, Phoenix, AZ, pp. 652-656.

[8] D. A. Pearce, A. G. Burr, and T. C. Tozer, "Comparison of countermeasures against slow Rayleigh fading for TDMA systems," in Colloquium on Advanced TDMA Techniques and Applications, IEEE, 1996, pp. $9 / 1-9 / 6$

[9] J. M. Torrance and L. Hanzo, "Upper bound performance of adaptive modulation in a slow Rayleigh fading channel," Electron. Lett., pp. 169-171, Apr. 1996.

[10] _ "Adaptive modulation in a slow Rayleigh fading channel," in Proc. 7th Personal, Indoor and Mobile Radio Communications (PIMRC) Conf., IEEE, 1996, pp. 497-501.

[11] _ "Optimization of switching levels for adaptive modulation in a slow Rayleigh fading channel." Electron. Lett., vol. 32, no. 13, pp. 1167-1169, June 20, 1996.

[12] _ "Demodulation level selection in adaptivemodulation," Electron. Lett., vol. 32, no. 19, pp. 1751-1752, Sept. 12, 1996.

[13] _ "Performance upper bound of adaptive QAM in slow Rayleighfading environments," in Proc. ICCS'96/ISPAC'96, Singapore, Westin, Nov. 25-29, 1996, pp. 1653-1657.

[14] M. Morimoto, H. Harada, M. Okada, and S. Komaki, "A study on power assignment of hierarchical modulation schemes for digital broadcasting," IEICE Trans. Commun., vol. 77, no. 12, pp. 1495-1500, 1994.

[15] J. Torrance and L. Hanzo, "Latency considerations for adaptive modulation in slow Rayleigh fading," in Proc. IEEE VTC'97, Phoenix, AZ, 1997, pp. 1204-1209. 
[16] _ _ "Statistical multiplexing for mitigating latency in adaptive modems," submitted to PIMRC'97, Helsinki, Finland, Sept. 1-4, 1997.

[17] W. C. Y. Lee, "Spectrum efficiency in cellular," IEEE Trans. Veh. Technol., vol. 38, pp. 69-75, Apr. 1989.

[18] W. H. Press, S. A. Teukolsky, W. T. Vetterling, and B. P. Flannery, Numerical Recipes in C. Cambridge, U.K.: Cambridge Univ. Press, 1994.

[19] M.-S. Alouini and A. Goldsmith, "Capacity of Nakagami multipath fading channels," in Proc. IEEE VTC'97, Phoenix, AZ, pp. 652-656.

[20] M. Andersin, "Real-time estimation of the signal to interference ratio in cellular radio systems," in Proc. VTC'97, Phoenix, AZ, pp. 1089-1093.

[21] A. Urie, M. Streeton, and C. Mourot, "An advanced TDMA mobile access system for UMTS," IEEE Commun. Mag., pp. 38-47, Feb. 1995.

[22] European RACE D731 Public Deliverable, "Mobile communication networks, general aspects and evolution," Sept. 1995.

[23] W. Webb, "Spectrum efficiency of multilevel modulation schemes in mobile radio communications," IEEE Trans. Commun., vol. 43, pp. 2344-2349, Aug. 1995.

[24] "Groupe Speciale Mobile (GSM) recommendation," Apr. 1988

[25] K. Fukawa and H. Suzuki, "Blind interference cancelling equalizer for mobile radio communications," IEICE Trans. Commun., vol. E77-B, no. 5, pp. 580-588, 1994.

[26] S. W. Wales, "Technique for cochannel interference suppression in TDMA mobile radio systems," Proc. Inst. Elec. Eng. Communications, vol. 142, no. 2, pp. 106-114, 1995.

[27] E. L. Kuan and L. Hanzo, "Joint detection CDMA techniques for thirdgeneration transceivers," in ACTS'98, Rhodos, Greece, June 1998, pp. 727-732.

[28] P. B. Hagerman, "Downlink relative co-channel interference power in cellular radio systems," in 45th Vehicular Technology Conf., IEEE, 1995 pp. 366-370.

[29] H. Murata, A. Fujiwara, and S. Yoshida, "Computational complexity reduction of trellis-coded co-channel interference canceller," IEICE Trans. Commun., vol. E79-B, no. 3, pp. 342-351, 1996.

[30] R. Berangi, P. Leung, and M. Faulkner, "Cochannel interfernce cancellation for mobile communication systems," in Proc. IEEE ICUPC Conf., IEEE, Sept. 27-29, 1996, pp. 438-442.

Jeff Torrance graduated in applied physics. He then spent two years with IBM developing DSP algorithms and was awarded the M.Sc. degree by research. He completed the Ph.D. degree in digital modulation with the Wireless Multimedia Communications Group at the University of Southampton (U.K.).

After receiving the Ph.D. degree, he started work for the Wireless Technology Practice at PA Consulting Group (Cambridge, U.K.). His main interests are transmission and reception for second- and third-generation mobile radio.

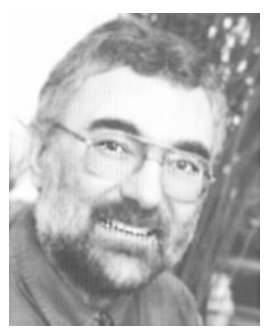

Lajos Hanzo (M'91-SM'92) received the Master's degree in electronics in 1976 and the Ph.D. degree in 1983, both from the Technical University of Budapest, Budapest, Hungary.

During his 23-year career in telecommunications he has held various research and academic posts in Hungary, Germany, and the U.K. Since 1986 he has been with the Department of Electronics and Computer Science, University of Southampton, U.K. and has been a Consultant to Multiple Access Communications Ltd., U.K. Currently he holds a Chair in Telecommunications. He coauthored three books on mobile radio communications, published over 200 research papers, organized and chaired conference sessions, presented overview lectures, and was awarded a number of distinctions. Currently he is managing an academic research team, working on a range of research projects in the field of wireless multimedia communications under the auspices of the Engineering and Physical Sciences Research Council (EPSRC), U.K., the European Advanced Communications Technologies and Services (ACTS) Programme, and the Mobile Virtual Centre of Excellence (VCE), U.K. As an enthusiastic supporter of Industry-Academia liaison, he offers a range of short courses on all aspects of Wireless Multimedia Communications. For further information on research in progress and associated publications please refer to the home page http://www-mobile.ecs.soton.ac.uk.

Thomas Keller studied electrical engineering at the University of Karlsruhe, Karlsruhe, Germany, Ecole Superieure d'Ingenieurs en Electronique et Electrotechnique, Paris, France, and the University of Southampton, U.K. He graduated with the Dipl.-Ing. degree in 1995. Since then he has been with the Wireless Multimedia Communications Group at the University of Southampton, working toward the Ph.D. degree in mobile communications.

His current areas of interest include adaptive OFDM transmission, wideband channel estimation, and error-correction coding. 